TRANSACTIONS OF THE

AMERICAN MATHEMATICAL SOCIETY

Volume 363, Number 8, August 2011, Pages 4401-4420

S 0002-9947(2011)05353-5

Article electronically published on March 7, 2011

\title{
ON THE BICANONICAL MORPHISM OF QUADRUPLE GALOIS CANONICAL COVERS
}

\author{
FRANCISCO JAVIER GALLEGO AND BANGERE P. PURNAPRAJNA
}

\begin{abstract}
In this article we study the bicanonical map $\varphi_{2}$ of quadruple Galois canonical covers $X$ of surfaces of minimal degree. We show that $\varphi_{2}$ has diverse behavior and exhibits most of the complexities that are possible for a bicanonical map of surfaces of general type, depending on the type of $X$. There are cases in which $\varphi_{2}$ is an embedding, and if it so happens, $\varphi_{2}$ embeds $X$ as a projectively normal variety, and there are cases in which $\varphi_{2}$ is not an embedding. If the latter, $\varphi_{2}$ is finite of degree 1,2 or 4 . We also study the canonical ring of $X$, proving that it is generated in degree less than or equal to 3 and finding the number of generators in each degree. For generators of degree 2 we find a nice general formula which holds for canonical covers of arbitrary degrees. We show that this formula depends only on the geometric and the arithmetic genus of $X$.
\end{abstract}

\section{INTRODUCTION}

Canonical covers of surfaces of minimal degree have a ubiquitous presence in diverse contexts in the geometry of surfaces and threefolds. For example they appear in the classification of surfaces of general type with small $c_{1}^{2}$ as shown in the work of Horikawa, and they play an important role in mapping the geography of surfaces of general type. They appear as unavoidable boundary cases in the study of linear series on Calabi-Yau threefolds as the works of Beltrametti and Szemberg (see BS00), Oguiso and Peternell (see OP95) and the authors (see GP98) show, and in the study of the canonical ring of a variety of general type as can be seen in the article of Green Gre82. They are also a useful source in constructing new examples of surfaces of general type.

Double and triple canonical covers were classified by Horikawa (see [Hor76]) and Konno (see Kon91]). In GP07 and GP08, the authors classified Galois canonical covers of degree 4 . The classification showed that quadruple canonical covers behave quite differently from canonical covers of all other degrees; for instance, quadruple canonical covers are the only covers that admit families with unbounded geometric

Received by the editors July 3, 2009 and, in revised form, February 9, 2010.

2000 Mathematics Subject Classification. Primary 14J10, 14J29.

Key words and phrases. Surfaces of general type, bicanonical map, quadruple Galois canonical covers, canonical ring, surfaces of minimal degree.

The first author was partly supported by Spanish Government grant MTM2006-04785 and by Complutense grant PR27/05-13876 and is part of the Complutense Research group 910772. He also thanks the Department of Mathematics of the University of Kansas for its hospitality.

The second author thanks the General Research Fund of Kansas for partly supporting this research project. He also thanks the Department of Algebra of the Universidad Complutense de Madrid for its hospitality. 
genus and families with unbounded irregularity. Hence, the geography of Chern numbers of quadruple canonical covers is much more complex.

One of the much studied objects for surfaces of general type is the bicanonical map. In this article we prove results on the bicanonical morphisms of quadruple canonical covers of surfaces of minimal degree. Our results show that the behavior of these bicanonical maps is quite generic, that is, it exhibits all the diversities and complexities that are possible for a bicanonical map of a surface of general type. All of this is amply illustrated in the following theorem:

Theorem 0.1. Let $\varphi: X \longrightarrow W$ be a quadruple Galois canonical cover of a surface $W$ of minimal degree. Then the bicanonical map $\varphi_{2}$ of $X$ is

1) a morphism which embeds $X$ as a projectively normal variety if $X$ is of Type 1, 2 or 3.2 (see Theorem 1.3 for the description of each type);

2) a birational morphism but not an embedding if $X$ is of Type 6, 7, 8 or 9;

3) a finite morphism of degree 2 if $X$ is of Type 3.1, 4 or 5.2;

4) a finite morphism of degree 4 if $X$ is of Type 5.1.

The diverse behavior of the bicanonical maps exhibited in the above theorem is not seen in canonical covers of lower degrees and conjecturally does not happen for covers of all other degrees.

In Section 2, we deal with those types of quadruple Galois canonical covers for which $2 K_{X}$ is very ample and embeds $X$ as a projectively normal surface. Other than covers of the projective plane, the manifestation of such behavior can be seen in surfaces $X$ with unbounded $p_{g}$ but with bounded $q$. However, in the case of families with unbounded $q, 2 K_{X}$ is not very ample, even though the image is a projectively normal variety. One of the frequently used techniques to show that a 2 -Veronese subring of a graded ring is generated in degree one is to first show that the ambient graded ring is generated in degree less than or equal to two. Formally one can construct graded rings in commutative algebra where this does not happen and yet the 2 -Veronese subring is generated in degree 1 . In this context, the quadruple covers of Types 1,2 and 3.2 provide natural examples where the canonical ring is not generated in degree less than or equal to two, yet the 2-Veronese subrings are generated in degree less than or equal to one, thereby showing the normal generation of the bicanonical map.

Section 3 deals with those quadruple covers for which bicanonical maps are finite and not birational. The types for which the bicanonical maps are not birational have unbounded $p_{g}$ and $q$. But the results of Xiao (see Xia90, Theorems 1, 2 and 3]) say that if the bicanonical maps are not birational, then they form bounded families with respect to $p_{g}$ unless the surfaces possess a genus 2 pencil. In Section 3 , we exhibit the genus 2 pencils that are indeed fibrations. So in addition to being a genus 3 fibration over $\mathbf{P}^{1}$, the families of Type 3.1 are also genus 2 fibrations over an elliptic curve, and the families of Type 4 and 5 are also genus 2 fibrations over a curve of genus $m$ and a curve of genus $m+1$, respectively, where $m$ takes on unbounded values.

Section 4 deals with the Types $6,7,8$ and 9 where the image of the canonical map $W$ is a singular surface of minimal degree. It was shown in [GP07] that in such a case $p_{g} \leq 4$ and $q=0$. The behavior of the bicanonical map is very interesting for these types of surfaces: it is always birational but never an embedding. More interestingly, it is birational in two different ways: for Types 6,7 or $9,\left|2 K_{X}\right|$ does not separate directions at the unique point $x \in \varphi^{-1}\{w\}$ (and is an isomorphism outside 
$x$. For Type $8,\left|2 K_{X}\right|$ does not separate the two points $x_{1}$ and $x_{2}$ of $\varphi^{-1}\{w\}$, although $\varphi_{2}$ is locally an embedding at both of them (and it is an isomorphism outside $x_{1}$ and $x_{2}$ ). We prove these results by proving a nonvanishing theorem for certain ideal sheaves. To accomplish this, we construct explicit factorizations of some birational maps which are in half the cases crepant and in the other half not and handle the question of the nonvanishing result on a suitably constructed desingularization of $X$. In all of this, the algebra structure of the map $p$ in (1.3.1) (the so-called desingularized diagram), which was precisely described in [GP07, also plays an important role.

The canonical ring of a surface of general type and the degrees of its generators have attracted interest among geometers for various reasons. One such reason is its applications to the study of Calabi-Yau threefolds. For example, results on ring generation are used in determining the very ampleness of line bundles on Calabi-Yau threefolds in the article [GP98] and have provided motivation in the construction of new examples of Calabi-Yau threefolds as can be seen in the work of Casnati Cas06. In Section 5 we prove a general result, Theorem 5.1, that gives a nice formula for the number of generators in degree 2 of the canonical ring of canonical covers, of arbitrary degree, of surfaces of minimal degree. The formula shows that the number of generators in degree 2 only depends on the geometric and arithmetic genus of $X$. Theorem 5.2, which determines the generators of the canonical ring of quadruple covers, shows that there is no such formula for generators of degree 3 of the canonical ring of $X$ if $X$ is an irregular surface of general type. In fact, Theorem 5.2 shows that this number depends on the algebra structure of $\varphi_{*} \mathscr{O}_{X}$.

\section{Preliminaries And notation}

We introduce the notation and the basic definitions that we will use throughout the article:

Convention. We work over an algebraically closed field of characteristic 0 .

Notation 1.1. Throughout this article, unless otherwise stated, we will make the following assumptions and use the following notation:

(1) $W$ will be an embedded projective algebraic surface of minimal degree, i.e., a surface such that $\operatorname{deg} W=\operatorname{codim} W+1$.

(2) $X$ will be a projective algebraic normal surface with at worst canonical singularities (that is, $X$ is smooth or has rational double points; see Bad01] for details about rational double points), whose canonical divisor $K_{X}$ is ample and base-point-free.

(3) We will denote the canonical map of $X$ as $\varphi$. Note that, by (2), $\varphi$ is in fact a finite morphism.

(4) We will assume $\varphi$ to be a Galois morphism of degree 4 whose image is a surface $W$ of minimal degree, that is, $\varphi: X \longrightarrow W$ will be a quadruple Galois canonical cover of a surface $W$ of minimal degree.

(5) We will denote the bicanonical map of $X$ as $\varphi_{2}$.

We also recall the following standard notation, which will also be used throughout the article:

(6) By $\mathbf{F}_{e}$ we denote the Hirzebruch surface whose minimal section has selfintersection $-e$. If $e>0$ let $C_{0}$ denote the minimal section of $\mathbf{F}_{e}$ and let $f$ 
be one of the fibers of $\mathbf{F}_{e}$. If $e=0, C_{0}$ will be a fiber of one of the families of lines and $f$ will be a fiber of the other family of lines of $\mathbf{F}_{0}$.

(7) If $a, b$ are integers such that $0<a \leq b$, consider two disjoint linear subspaces $\mathbf{P}^{a}$ and $\mathbf{P}^{b}$ of $\mathbf{P}^{a+b+1}$. We denote by $S(a, b)$ the smooth rational normal scroll obtained by joining corresponding points of a rational normal curve in $\mathbf{P}^{a}$ and a rational normal curve of $\mathbf{P}^{b}$. Recall that $S(a, b)$ is the image of $\mathbf{F}_{e}$ by the embedding induced by the complete linear series $\left|C_{0}+m f\right|$, with $a=m-e, b=m$ and $m \geq e+1$.

If $a=b$, the linear series $\left|m C_{0}+f\right|$ also gives a minimal degree embedding of $\mathbf{F}_{0}$, equivalent to the previous one by the automorphism of $\mathbf{P}^{1} \times \mathbf{P}^{1}=\mathbf{F}_{0}$ swapping the factors. In this case our convention will always be to choose $C_{0}$ and $f$ so that, when $W=S(m, m), W$ is embedded by $\left|C_{0}+m f\right|$.

If in addition $m=1, C_{0}$ and $f$ are indistinguishable in both $\mathbf{F}_{0}$ and $S(1,1)$, so, in such a case, for us $C_{0}$ will denote the fiber of any of the families of lines of $\mathbf{F}_{0}$ and $f$ will denote the fiber of the other family.

(8) If $b$ is an integer, $b>1$, consider a linear subspace $\mathbf{P}^{b}$ of $\mathbf{P}^{b+1}$. We denote by $S(0, b)$ the cone in $\mathbf{P}^{b+1}$ over a rational normal curve of $\mathbf{P}^{b}$. Recall that $S(0, b)$ is the image of $\mathbf{F}_{e}$ by the morphism induced by the complete linear series $\left|C_{0}+m f\right|$, with $b=m=e$ and hence $e>1$.

Remark 1.2. If $X \stackrel{\varphi}{\longrightarrow} W$ is a Galois cover and $W$ is smooth, then $\varphi$ is flat.

We now recall the main features of the classification of $\varphi$, which was obtained in GP08, Theorem 0.1] and in the main theorem of [GP07] (beware that the notation for the branch divisors used in Theorem 1.3 differs from the notation in GP08. when $G=\mathbf{Z}_{4}$ ). According to this classification, $\varphi$ falls into several different types described in the tables of the theorem below. We will refer to these types throughout the article.

Theorem 1.3. Let $\varphi: X \longrightarrow W$ be as in Notation 1.1 (i.e., let $\varphi$ be a quadruple Galois canonical cover of a surface $W$ of minimal degree).

1) If $W$ is smooth, then $W$ is either linear $\mathbf{P}^{2}$ or a smooth Hirzebruch surface $\mathbf{F}_{e}$, with $0 \leq e \leq 2$, embedded by $|H|$, where $H=C_{0}+m f(m \geq e+1)$. Recall that the Galois group $G$ of $\varphi$ is either $\mathbf{Z}_{4}$ or $\mathbf{Z}_{2}^{\oplus 2}$.

The morphism $\varphi$ is the composition of two double covers $X_{1} \stackrel{p_{1}}{\longrightarrow} W$ and $X \stackrel{p_{2}}{\longrightarrow} X_{1}$; the cover $p_{1}$ is branched along a divisor $D_{2}$ and the cover $p_{2}$ is branched along the $p_{1}^{*} D_{1}$, where $D_{1}$ is a divisor on $W$.

Moreover $\varphi$ fits into one of the following types, determined by these characteristics:

\begin{tabular}{|r|c|c|c|c|c|c|}
\hline Type & $W$ & $p_{g}(X)$ & $G$ & $D_{1} \sim$ & $D_{2} \sim$ & $q(X)$ \\
\hline 1 & $\mathbf{P}^{2}$ & 3 & $\mathbf{Z}_{4}, \mathbf{Z}_{2}^{\oplus 2}$ & quartic & quartic & 0 \\
\hline 2 & $S(m, m-e)$ & $2 m-e+2$ & $\mathbf{Z}_{4}, \mathbf{Z}_{2}^{\oplus 2}$ & $2 C_{0}+(2 m+2) f$ & $4 C_{0}+(2 e+2) f$ & 0 \\
\hline 3.1 & $S(1,1)$ & 4 & $\mathbf{Z}_{4}, \mathbf{Z}_{2}^{\oplus 2}$ & $2 C_{0}+6 f$ & $4 C_{0}$ & 1 \\
\hline 3.2 & $S(m, m),(m>1)$ & $2 m+2$ & $\mathbf{Z}_{4}, \mathbf{Z}_{2}^{\oplus 2}$ & $2 C_{0}+(2 m+4) f$ & $4 C_{0}$ & 1 \\
\hline 4 & $S(m, m)$ & $2 m+2$ & $\mathbf{Z}_{2}^{\oplus 2}$ & $6 C_{0}+2 f$ & $(2 m+2) f$ & $m$ \\
\hline 5.1 & $S(1,1)$ & 4 & $\mathbf{Z}_{2}^{\oplus 2}$ & $6 f$ & $6 C_{0}$ & 4 \\
\hline 5.2 & $S(m, m),(m>1)$ & $2 m+2$ & $\mathbf{Z}_{2}^{\oplus 2}$ & $(2 m+4) f$ & $6 C_{0}$ & $m+3$ \\
\hline
\end{tabular}


2) If $W$ is not smooth, then $W=S(0,2)$ (a quadric cone in $\mathbf{P}^{3}$ ), $X$ is regular and $\varphi: X \longrightarrow W$ fits into a commutative diagram (see [GP07]):

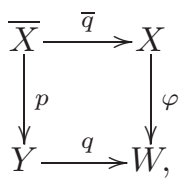

where $q$ is the minimal desingularization of $W, \bar{X}$ is the normalization of the reduced part of $X \times_{W} Y$ and has at worst canonical singularities, and $p$ and $\bar{q}$ are induced by the projections from the fiber product onto each factor, $\bar{q}$ being the morphism from $\bar{X}$ to its canonical model $X$. The structure of $p$ is similar to the structure of $\varphi$ described above for $W$ smooth (see the main theorem of GP07] for details) and $\varphi$ can be classified in another four types, determined by the following properties:

\begin{tabular}{|c|c|c|c|c|c|}
\hline Type & $W$ & $p_{g}(X)$ & $G$ & $\bar{q}$ is & $q(X)$ \\
\hline
\end{tabular}

\begin{tabular}{|c|c|c|c|c|c|}
\hline 6 & $S(0,2)$ & 4 & $\mathbf{Z}_{2}^{\oplus 2}$ & crepant & 0 \\
\hline 7 & $S(0,2)$ & 4 & $\mathbf{Z}_{4}$ & crepant & 0 \\
\hline 8 & $S(0,2)$ & 4 & $\mathbf{Z}_{2}^{\oplus 2}$ & not crepant & 0 \\
\hline 9 & $S(0,2)$ & 4 & $\mathbf{Z}_{4}$ & not crepant & 0 \\
\hline
\end{tabular}

\section{Normal Generation of the BicAnonical BUndLe}

In this section we study the types of quadruple Galois canonical covers $X$ for which $2 K_{X}$ is normally generated, i.e., we find out for what $X$ the bicanonical morphism $\varphi_{2}$ embeds $X$ as a projectively normal variety. As we will see in Section 4 . $\varphi_{2}$ is never an embedding if $W$ is singular, so throughout this section we assume $W$ to be smooth.

Recall that by Remark 1.2, since we are assuming $W$ to be smooth, $\varphi$ is flat. Furthermore, the pushdown of $\mathscr{O}_{X}$ to $W$ splits as an $\mathscr{O}_{W}$-module as follows:

$$
\varphi_{*} \mathscr{O}_{X}=\mathscr{O}_{W} \oplus L_{1}^{*} \oplus L_{2}^{*} \oplus L_{3}^{*},
$$

with $L_{1}, L_{2}$ and $L_{3}$ and $D_{1}$ and $D_{2}$ of Theorem 1.3 satisfying the following properties (see GP08, Remark 3.1], where the multiplicative structure that turns the second term of (2.0.2) into an $\mathscr{O}_{W}$-algebra is also described):

$$
L_{1} \otimes L_{2}=L_{3} \text {, where } L_{1}^{*}=\mathscr{O}_{W}\left(-1 / 2 D_{2}\right) \text { and } L_{2}^{*}=\mathscr{O}_{W}\left(-1 / 2 D_{1}\right),
$$

We will keep the notation introduced in (2.0.2) and (2.0.3) for the remainder of the article.

To prove the normal generation of $2 K_{X}$ we will look at multiplication maps of global sections of line bundles on $X$. To study these maps we will use the $\mathscr{O}_{W^{-}}$ algebra structure of $\varphi_{*} \mathscr{O}_{X}$ as the following lemma explains:

Lemma 2.1. Let $A_{1}, A_{2}$ be two line bundles on $W$ and let $M_{1}=\varphi^{*} A_{1}$ and $M_{2}=\varphi^{*} A_{2}$ be their pullbacks on $X$. Let

$$
H^{0}\left(M_{1}\right) \otimes H^{0}\left(M_{2}\right) \stackrel{\beta}{\longrightarrow} H^{0}\left(M_{1} \otimes M_{2}\right)
$$


be the multiplication map of global sections of $M_{1}$ and $M_{2}$ and let

$$
\begin{array}{ccc}
H^{0}\left(A_{1}\right) \otimes H^{0}\left(A_{2}\right) & \stackrel{\beta_{1}}{\longrightarrow} \quad H^{0}\left(A_{1} \otimes A_{2}\right), \\
H^{0}\left(A_{1}\right) \otimes H^{0}\left(A_{2} \otimes L_{1}^{*}\right) & \stackrel{\beta_{2}}{\longrightarrow} & H^{0}\left(A_{1} \otimes A_{2} \otimes L_{1}^{*}\right), \\
H^{0}\left(A_{1}\right) \otimes H^{0}\left(A_{2} \otimes L_{2}^{*}\right) & \stackrel{\beta_{3}}{\longrightarrow} & H^{0}\left(A_{1} \otimes A_{2} \otimes L_{2}^{*}\right), \\
H^{0}\left(A_{1} \otimes L_{1}^{*}\right) \otimes H^{0}\left(A_{2} \otimes L_{2}^{*}\right) & \stackrel{\beta_{4}}{\longrightarrow} & H^{0}\left(A_{1} \otimes A_{2} \otimes L_{3}^{*}\right)
\end{array}
$$

be multiplication maps of global sections of line bundles on $W$.

1) If $\beta_{1}, \beta_{2}, \beta_{3}$ and $\beta_{4}$ are surjective, so is $\beta$.

2) If $H^{0}\left(A_{1} \otimes A_{2} \otimes L_{3}^{*}\right) \neq 0$ but $H^{0}\left(A_{i} \otimes L_{j}^{*}\right)=0$ for either

$$
\begin{aligned}
& i=1 \text { and } j=1,2 \text {, or } \\
& i=2 \text { and } j=1,2 \text {, or } \\
& i=1,2 \text { and } j=1 \text {, or } \\
& i=1,2 \text { and } j=2,
\end{aligned}
$$

then $\beta$ is not surjective.

Proof. We have by projection formula

$$
\begin{aligned}
H^{0}\left(\varphi_{*} M_{i}\right)= & H^{0}\left(A_{i}\right) \oplus H^{0}\left(A_{i} \otimes L_{1}^{*}\right) \oplus H^{0}\left(A_{i} \otimes L_{2}^{*}\right) \oplus H^{0}\left(A_{i} \otimes L_{3}^{*}\right), \\
H^{0}\left(\varphi_{*}\left(M_{1} \otimes M_{2}\right)\right)= & H^{0}\left(A_{1} \otimes A_{2}\right) \oplus H^{0}\left(A_{1} \otimes A_{2} \otimes L_{1}^{*}\right) \\
& \oplus H^{0}\left(A_{1} \otimes A_{2} \otimes L_{2}^{*}\right) \oplus H^{0}\left(A_{1} \otimes A_{2} \otimes L_{3}^{*}\right) .
\end{aligned}
$$

The surjectivity of $\beta$ is equivalent to the surjectivity of

$$
H^{0}\left(\varphi_{*} M_{1}\right) \otimes H^{0}\left(\varphi_{*} M_{2}\right) \stackrel{\beta^{\prime}}{\longrightarrow} H^{0}\left(\varphi_{*}\left(M_{1} \otimes M_{2}\right)\right) .
$$

The $\mathscr{O}_{W}$-algebra structure of $\varphi_{*} \mathscr{O}_{X}$ is given by a multiplication map

$$
\varphi_{*} \mathscr{O}_{X} \otimes \varphi_{*} \mathscr{O}_{X} \longrightarrow \varphi_{*} \mathscr{O}_{X}
$$

which splits in several summands according to (2.0.2), as explained in GP08, Remark 3.1]. From them, we are interested in the following four:

$$
\begin{array}{cc}
\mathscr{O}_{W} \otimes \mathscr{O}_{W} & \stackrel{\simeq}{\longrightarrow} \mathscr{O}_{W}, \\
\mathscr{O}_{W} \otimes L_{1}^{*} & \stackrel{\simeq}{\longrightarrow} L_{1}^{*}, \\
\mathscr{O}_{W} \otimes L_{2}^{*} & \stackrel{\simeq}{\longrightarrow} L_{2}^{*}, \\
L_{1}^{*} \otimes L_{2}^{*} & \stackrel{\simeq}{\longrightarrow} L_{3}^{*} .
\end{array}
$$

The map $\beta^{\prime}$ also splits according to (2.1.1), so (2.1.2) implies that if $\beta_{1}, \beta_{2}, \beta_{3}$ and $\beta_{4}$ surject, all the summands of $H^{0}\left(\varphi_{*}\left(M_{1} \otimes M_{2}\right)\right)$ described in (2.1.1) are in the image of $\beta^{\prime}$, so $\beta^{\prime}$ and hence $\beta$ are surjective. On the other hand, GP08, Remark 3.1] also tells us that the last map of (2.1.2) is the only summand mapping to $L_{3}$. Thus, under the hypotheses of 2$), H^{0}\left(A_{1} \otimes A_{2} \otimes L_{3}^{*}\right) \neq 0$ but it is not in the image of $\beta^{\prime}$, so $\beta^{\prime}$ and hence $\beta$ are not surjective in this case.

To apply Lemma 2.1 in proving the normal generation of $2 K_{X}$ we will need this easy but useful observation that helps to handle multiplication maps of global sections. 
Observation 2.2. Let $E$ and $\mathscr{F}_{1}, \ldots, \mathscr{F}_{n}$ be coherent sheaves on a variety $X$. Consider the map $H^{0}(E) \otimes H^{0}\left(\mathscr{F}_{1} \otimes \cdots \otimes \mathscr{F}_{r}\right) \stackrel{\psi}{\longrightarrow} H^{0}\left(E \otimes \mathscr{F}_{1} \otimes \cdots \otimes \mathscr{F}_{r}\right)$ and the maps

$$
\begin{aligned}
& H^{0}(E) \otimes H^{0}\left(\mathscr{F}_{1}\right) \stackrel{\alpha_{1}}{\longrightarrow} H^{0}\left(E \otimes \mathscr{F}_{1}\right), \\
& H^{0}\left(E \otimes \mathscr{F}_{1}\right) \otimes H^{0}\left(\mathscr{F}_{2}\right) \stackrel{\alpha_{2}}{\longrightarrow} H^{0}\left(E \otimes \mathscr{F}_{1} \otimes \mathscr{F}_{2}\right), \\
& \quad \cdots \cdots \\
& H^{0}\left(E \otimes \mathscr{F}_{1} \otimes \cdots \otimes \mathscr{F}_{r-1}\right) \otimes H^{0}\left(\mathscr{F}_{r}\right) \stackrel{\alpha_{r}}{\longrightarrow} H^{0}\left(E \otimes \mathscr{F}_{1} \otimes \cdots \otimes \mathscr{F}_{r}\right) .
\end{aligned}
$$

If $\alpha_{1}, \ldots, \alpha_{r}$ are surjective, then $\psi$ is surjective.

For the reader's convenience we also state here the following generalization by Mumford of a lemma of Castelnuovo, which we will use throughout the article to help us prove the surjectivity of certain multiplication maps of global sections.

Theorem 2.3. Let $\mathscr{L}$ be a base-point-free line bundle on a variety $X$ and let $\mathscr{F}$ be a coherent sheaf on $X$. If $H^{i}\left(\mathscr{F} \otimes \mathscr{L}^{-i}\right)=0$ for all $i \geq 1$, then the multiplication map

$$
H^{0}\left(\mathscr{F} \otimes \mathscr{L}^{\otimes i}\right) \otimes H^{0}(\mathscr{L}) \rightarrow H^{0}\left(\mathscr{F} \otimes \mathscr{L}^{\otimes i+1}\right)
$$

is surjective for all $i \geq 0$.

Proof. See Mum70, p. 41, Theorem 2]. Note that the assumption made there of $\mathscr{L}$ being ample is unnecessary.

Now we are ready to prove the normal generation of $2 K_{X}$ for surfaces $X$ of Types 1, 2 and 3.2. As a warm-up for the rest, we start with the simplest case, that is, the case when $W=\mathbf{P}^{2}$ :

Theorem 2.4. Let $\varphi: X \longrightarrow W$ be a quadruple Galois canonical cover of Type 1 . Then $2 K_{X}$ is very ample and $\left|2 K_{X}\right|$ embeds $X$ as a projectively normal variety.

Proof. Recall that $\mathscr{O}_{X}\left(K_{X}\right)=\varphi^{*} \mathscr{O}_{\mathbf{P}^{2}}(1)$. Since $K_{X}$ is ample, the normal generation of $2 K_{X}$ is equivalent to the surjectivity of

$$
H^{0}\left(\mathscr{O}_{X}\left(2 K_{X}\right)\right) \otimes H^{0}\left(\mathscr{O}_{X}\left(2 n K_{X}\right)\right) \longrightarrow H^{0}\left(\mathscr{O}_{X}\left((2 n+2) K_{X}\right)\right),
$$

for all $n \geq 1$. We prove it in two steps.

Step 1. The first step is to show that

$$
H^{0}\left(\mathscr{O}_{X}\left(2 K_{X}\right)\right) \otimes H^{0}\left(\mathscr{O}_{X}\left(2 K_{X}\right)\right) \stackrel{\beta}{\longrightarrow} H^{0}\left(\mathscr{O}_{X}\left(4 K_{X}\right)\right)
$$

surjects. We know (see the structure of $\varphi$ as described in Theorem 1.3) that, for Type 1 ,

$$
\varphi_{*} \mathscr{O}_{X}=\mathscr{O}_{\mathbf{P}^{2}} \oplus \mathscr{O}_{\mathbf{P}^{2}}(-2) \oplus \mathscr{O}_{\mathbf{P}^{2}}(-2) \oplus \mathscr{O}_{\mathbf{P}^{2}}(-4)
$$

hence we have

$$
\begin{aligned}
& H^{0}\left(\varphi_{*} \mathscr{O}_{X}\left(2 K_{X}\right)\right)=H^{0}\left(\mathscr{O}_{\mathbf{P}^{2}}(2)\right) \oplus H^{0}\left(\mathscr{O}_{\mathbf{P}^{2}}\right) \oplus H^{0}\left(\mathscr{O}_{\mathbf{P}^{2}}\right) \quad \text { and } \\
& H^{0}\left(\varphi_{*} \mathscr{O}_{X}\left(4 K_{X}\right)\right)=H^{0}\left(\mathscr{O}_{\mathbf{P}^{2}}(4)\right) \oplus H^{0}\left(\mathscr{O}_{\mathbf{P}^{2}}(2)\right) \oplus H^{0}\left(\mathscr{O}_{\mathbf{P}^{2}}(2)\right) \oplus H^{0}\left(\mathscr{O}_{\mathbf{P}^{2}}\right)
\end{aligned}
$$


Then Lemma 2.1, 1) tells us that in order to prove the surjectivity of $\beta$ it is enough to show the surjectivity of the following multiplication maps on $W$ :

$$
\begin{array}{rll}
H^{0}\left(\mathscr{O}_{\mathbf{P}^{2}}(2)\right) \otimes H^{0}\left(\mathscr{O}_{\mathbf{P}^{2}}(2)\right) & \stackrel{\beta_{1}}{\longrightarrow} & H^{0}\left(\mathscr{O}_{\mathbf{P}^{2}}(4)\right), \\
H^{0}\left(\mathscr{O}_{\mathbf{P}^{2}}\right) \otimes H^{0}\left(\mathscr{O}_{\mathbf{P}^{2}}(2)\right) & \stackrel{\beta_{2}}{\longrightarrow} & H^{0}\left(\mathscr{O}_{\mathbf{P}^{2}}(2)\right), \\
H^{0}\left(\mathscr{O}_{\mathbf{P}^{2}}\right) \otimes H^{0}\left(\mathscr{O}_{\mathbf{P}^{2}}\right) & \stackrel{\beta_{4}}{\longrightarrow} & H^{0}\left(\mathscr{O}_{\mathbf{P}^{2}}\right) .
\end{array}
$$

The surjectivity of $\beta_{1}$ follows from the projective normality of the Veronese surface, and the surjectivity of $\beta_{2}$ and $\beta_{4}$ is trivial.

Step 2. To complete the proof, we need to show that the multiplication map (2.4.1) surjects for all $n \geq 2$. In view of Observation 2.2 it is enough to show that

$$
H^{0}\left(\mathscr{O}_{X}\left(n^{\prime} K_{X}\right)\right) \otimes H^{0}\left(\mathscr{O}_{X}\left(K_{X}\right)\right) \longrightarrow H^{0}\left(\mathscr{O}_{X}\left(\left(n^{\prime}+1\right) K_{X}\right)\right)
$$

for all $n^{\prime} \geq 4$. Since $K_{X}$ is ample and base-point-free, this follows from Theorem 2.3 and the Kawamata-Viehweg vanishing theorem.

Now we go on to study the normal generation of $2 K_{X}$ for those remaining cases for which $W$ is smooth. In these cases $W$ is a Hirzebruch surface, so in order to apply Lemma 2.1 we first need to know the surjectivity of certain multiplication maps of global sections of line bundles on Hirzebruch surfaces. This is done in the next lemma, where we give sufficient conditions for the surjectivity of such maps.

Lemma 2.5. Let $W=\mathbf{F}_{e}, e=0,1$ or 2 . Let $\mathscr{L}_{1}=\mathscr{O}_{W}\left(a_{1} C_{0}+b_{1} f\right)$ and $\mathscr{L}_{2}=$ $\mathscr{O}_{W}\left(a_{2} C_{0}+b_{2} f\right)$ be two line bundles on $W$, with $a_{i} \geq 0, b_{i} \geq a_{i} e$. Then the multiplication map

$$
H^{0}\left(\mathscr{L}_{1}\right) \otimes H^{0}\left(\mathscr{L}_{2}\right) \stackrel{\mu}{\longrightarrow} H^{0}\left(\mathscr{L}_{1} \otimes \mathscr{L}_{2}\right)
$$

is surjective if, in addition, $\mathscr{L}_{1}$ and $\mathscr{L}_{2}$ satisfy one of the following conditions:

(a) $a_{1} \geq 1, a_{2}=n a_{1}, b_{2}=n b_{1}$ with $n \geq 1$, and $b_{1}>a_{1} e$;

(b) $a_{1} \geq 1$ and $b_{2}-b_{1} \geq\left(a_{2}-a_{1}\right) e-1$ and, further, $a_{2} \geq 2 a_{1}-2+e$ when $e \geq 1$, and $a_{2} \geq 2 a_{1}-1$ when $e=0 ;$

(c) $a_{1}>0, a_{2}=0$;

(d) $a_{1}=a_{2}=1$;

(e) $W=\mathbf{F}_{0}$.

Proof. Observe that $\mathscr{L}_{1}$ and $\mathscr{L}_{2}$ are base-point-free because $a_{i} \geq 0, b_{i} \geq a_{i} e$.

We start proving the lemma under the assumption that (a) is satisfied. Observe that $\mathscr{L}_{1}$ is ample because $a_{1} \geq 1$ and $b_{1}>a_{1} e$ by hypothesis. Then (a) is equivalent to the normal generation of $\mathscr{L}_{1}$. We will apply [GP01, Theorem 1.3] for property $N_{0}$. Then we just need to show that $-K_{W} \cdot\left(a_{1} C_{0}+b_{1} f\right)=\left(2 C_{0}+(2+e) f\right) \cdot\left(a_{1} C_{0}+b_{1} f\right) \geq$ 3 . This amounts to showing $-a_{1} e+2 a_{1}+2 b_{1} \geq 3$, which is true by hypothesis. This proves (a).

To show the lemma when (b) is satisfied, we use Theorem 2.3 . Then it would be enough to show that $H^{1}\left(\mathscr{L}_{2} \otimes \mathscr{L}_{1}^{*}\right)=H^{1}\left(\mathscr{O}_{W}\left(\left(a_{2}-a_{1}\right) C_{0}+\left(b_{2}-b_{1}\right) f\right)\right)$ and $H^{2}\left(\mathscr{L}_{2} \otimes \mathscr{L}_{1}^{-2}\right)=H^{2}\left(\mathscr{O}_{W}\left(\left(a_{2}-2 a_{1}\right) C_{0}+\left(b_{2}-2 b_{1}\right) f\right)\right)$ both vanish. Let $\pi: W \longrightarrow$ $\mathbf{P}^{1}$ be the projection from $W$ to $\mathbf{P}^{1}$. Note that by hypothesis $a_{2}-a_{1} \geq 0$, and $a_{2}-2 a_{1} \geq-1$, which implies $R^{1} \pi_{*}\left(\mathscr{L}_{2} \otimes \mathscr{L}_{1}^{*}\right)=0$ and $R^{1} \pi_{*}\left(\mathscr{L}_{2} \otimes \bar{L}_{1}^{-2}\right)=0$. So $H^{j}\left(\mathscr{L}_{2} \otimes \mathscr{L}_{1}^{-j}\right)=H^{j}\left(\pi_{*}\left(\mathscr{L}_{2} \otimes \mathscr{L}_{1}^{-j}\right)\right)$ for $j=1$, 2. Since $a_{2}-a_{1} \geq 0$, then $\pi_{*}\left(\mathscr{L}_{2} \otimes \mathscr{L}_{1}^{*}\right)$ splits as the sum of $a_{2}-a_{1}+1$ line bundles as follows:

$$
\pi_{*}\left(\mathscr{L}_{2} \otimes \mathscr{L}_{1}^{*}\right)=\mathscr{O}_{\mathbf{P}^{1}}\left(b_{2}-b_{1}\right) \oplus \cdots \oplus \mathscr{O}_{\mathbf{P}^{1}}\left(b_{2}-b_{1}-\left(a_{2}-a_{1}\right) e\right) .
$$


Since by hypothesis $b_{2}-b_{1} \geq\left(a_{2}-a_{1}\right) e-1, H^{1}\left(\pi_{*}\left(\mathscr{L}_{2} \otimes \mathscr{L}_{1}^{*}\right)\right)$, and hence $H^{1}\left(\mathscr{L}_{2} \otimes \mathscr{L}_{1}^{*}\right)$, vanish. Since a curve has no second cohomology, $H^{2}\left(\mathscr{L}_{2} \otimes \mathscr{L}_{1}^{-2}\right)$ also vanishes. This proves the lemma if (b) holds.

Now assume (c) holds. We will use Observation 2.2 repeatedly and apply Theorem 2.3. By Observation 2.2 it suffices to prove that the multiplication map

$$
H^{0}\left(\mathscr{O}_{W}\left(a_{1} C_{0}+b_{1}^{\prime} f\right)\right) \otimes H^{0}\left(\mathscr{O}_{W}(f)\right) \stackrel{\mu^{\prime}}{\longrightarrow} H^{0}\left(\mathscr{O}_{W}\left(a_{1} C_{0}+\left(b_{1}^{\prime}+1\right) f\right)\right)
$$

is surjective for all $b_{1}^{\prime} \geq b_{1}$. Now, since $f$ is base-point-free, Theorem 2.3 tells us that, to prove that $\mu^{\prime}$ is surjective, it is enough to show $H^{1}\left(\mathscr{O}_{W}\left(a_{1} C_{0}+\left(b_{1}^{\prime}-1\right) f\right)\right)=$ 0 and $H^{2}\left(\mathscr{O}_{W}\left(a_{1} C_{0}+\left(b_{1}^{\prime}-2\right) f\right)\right)=0$. Since $a_{1}>0, R^{1} \pi_{*}\left(\mathscr{O}_{W}\left(a_{1} C_{0}+\left(b_{1}^{\prime}-1\right) f\right)\right)$ and $R^{1} \pi_{*}\left(\mathscr{O}_{W}\left(a_{1} C_{0}+\left(b_{1}^{\prime}-2\right) f\right)\right)$ both vanish and hence it is enough to prove the vanishing of $H^{1}\left(\pi_{*}\left(\mathscr{O}_{W}\left(a_{1} C_{0}+\left(b_{1}^{\prime}-1\right) f\right)\right)\right)$ and $H^{2}\left(\pi_{*}\left(\mathscr{O}_{W}\left(a_{1} C_{0}+\left(b_{1}^{\prime}-2\right) f\right)\right)\right)$. The latter cohomology vanishes because a curve has no second cohomology, and the former vanishes arguing as in (2.5.1) because $b_{1}^{\prime}-1-a_{1} e \geq b_{1}-1-a_{1} e \geq-1$. This settles (c).

Without loss of generality we may assume $b_{2} \geq b_{1}-2$ by interchanging $\mathscr{L}_{1}$ and $\mathscr{L}_{2}$. If (d) holds, the lemma follows directly from Theorem 2.3 arguing as for (b) or (c), taking into account that $R^{1} \pi_{*}\left(\mathscr{O}_{W}\left(\left(b_{2}-b_{1}\right) f\right)\right)$ and $R^{1} \pi_{*} \mathscr{O}_{W}\left(\left(-C_{0}+\left(b_{2}-\right.\right.\right.$ $\left.\left.2 b_{1}\right) f\right)$ ) vanish and that $b_{2}-b_{1} \geq-1$ by hypothesis.

We will now assume that (e) holds. Without loss of generality we may assume $a_{1}>0$ since in this case $W=\mathbf{F}_{0}$ and by an involution of $\mathbf{F}_{0}$ we can interchange $C_{0}$ and $f$ and rename $a_{2}$ as $a_{1}$ if need be. In view of Observation 2.2 it is enough to show that the multiplication maps

$$
\begin{array}{cccc}
H^{0}\left(\mathscr{O}_{W}\left(a_{1}^{\prime} C_{0}+b_{1}^{\prime} f\right)\right) \otimes H^{0}\left(\mathscr{O}_{W}(f)\right) & \stackrel{\mu_{1}}{\longrightarrow} & H^{0}\left(\mathscr{O}_{W}\left(a_{1}^{\prime} C_{0}+\left(b_{1}^{\prime}+1\right) f\right)\right) & \text { and } \\
H^{0}\left(\mathscr{O}_{W}\left(a_{1}^{\prime} C_{0}+b_{1}^{\prime} f\right)\right) \otimes H^{0}\left(\mathscr{O}_{W}\left(C_{0}\right)\right) & \stackrel{\mu_{2}}{\longrightarrow} & H^{0}\left(\mathscr{O}_{W}\left(\left(a_{1}^{\prime}+1\right) C_{0}+b_{1}^{\prime} f\right)\right) &
\end{array}
$$

are surjective for all $a_{1}^{\prime} \geq a_{1}$ and all $b_{1}^{\prime} \geq b_{1}$. The maps $\mu_{1}$ are surjective because (c) holds in this case. The maps $\mu_{2}$ are also surjective because (c) also holds if $b_{1}>0$, after applying an involution of $\mathbf{F}_{0}$ interchanging $C_{0}$ and $f$. Finally, if $b_{1}=0$, after the involution of $\mathbf{F}_{0}, \mu_{2}$ becomes

$$
H^{0}\left(\mathscr{O}_{W}\left(a_{1}^{\prime} f\right)\right) \otimes H^{0}\left(\mathscr{O}_{W}(f)\right) \stackrel{\mu_{2}}{\longrightarrow} H^{0}\left(\mathscr{O}_{W}\left(\left(a_{1}^{\prime}+1\right) f\right)\right),
$$

so its surjectivity follows from the surjectivity of multiplication maps of global sections on $\mathbf{P}^{1}$.

Now we turn our attention to the bicanonical morphism of quadruple Galois canonical covers $X$ of smooth rational normal scrolls when $X$ is regular.

Theorem 2.6. Let $\varphi: X \longrightarrow W$ be a quadruple Galois canonical cover of Type 2. Then $2 K_{X}$ is very ample and $\left|2 K_{X}\right|$ embeds $X$ as a projectively normal variety.

Proof. Recall that $K_{X}=\varphi^{*}\left(C_{0}+m f\right)$ (see Theorem 1.3). As in the proof of Theorem 2.4 since $K_{X}$ is ample, the normal generation of $2 K_{X}$ is equivalent to the surjectivity of

$$
H^{0}\left(\mathscr{O}_{X}\left(2 K_{X}\right)\right) \otimes H^{0}\left(\mathscr{O}_{X}\left(2 n K_{X}\right)\right) \longrightarrow H^{0}\left(\mathscr{O}_{X}\left((2 n+2) K_{X}\right)\right)
$$

for all $n \geq 1$. We prove this in two steps.

Step 1. The first step is to show that

$$
H^{0}\left(\mathscr{O}_{X}\left(2 K_{X}\right)\right) \otimes H^{0}\left(\mathscr{O}_{X}\left(2 K_{X}\right)\right) \stackrel{\beta}{\longrightarrow} H^{0}\left(\mathscr{O}_{X}\left(4 K_{X}\right)\right)
$$


surjects, and we argue as in Step 1 of the proof of Theorem 2.4. We know (see the structure of $\varphi$ as described in Theorem 1.3) that, for Type 2,

$\varphi_{*} \mathscr{O}_{X}=\mathscr{O}_{W} \oplus \mathscr{O}_{W}\left(-C_{0}-(m+1) f\right) \oplus \mathscr{O}_{W}\left(-2 C_{0}-(e+1) f\right) \oplus \mathscr{O}_{W}\left(-3 C_{0}-(m+e+2) f\right) ;$

hence we have

$$
\begin{aligned}
H^{0}\left(\varphi_{*} \mathscr{O}_{X}\left(2 K_{X}\right)\right)= & H^{0}\left(\mathscr{O}_{W}\left(2 C_{0}+2 m f\right)\right) \oplus H^{0}\left(\mathscr{O}_{W}\left(C_{0}+(m-1) f\right)\right) \\
& \oplus H^{0}\left(\mathscr{O}_{W}((2 m-e-1) f)\right)
\end{aligned}
$$

and

$$
\begin{aligned}
H^{0}\left(\varphi_{*} \mathscr{O}_{X}\left(4 K_{X}\right)\right)= & H^{0} \mathscr{O}_{W}\left(\left(4 C_{0}+4 m f\right)\right) \oplus H^{0}\left(\mathscr{O}_{W}\left(3 C_{0}+(3 m-1) f\right)\right) \\
& \oplus H^{0}\left(\mathscr{O}_{W}\left(\left(2 C_{0}+(4 m-e-1) f\right)\right)\right. \\
& \oplus H^{0}\left(\mathscr{O}_{W}\left(C_{0}+(3 m-e-2) f\right)\right) .
\end{aligned}
$$

Then Lemma 2.1, 1) tells us that in order to prove the surjectivity of $\beta$ it is enough to show the surjectivity of the following multiplication maps on $W$ :

$$
\begin{array}{r}
H^{0}\left(\mathscr{O}_{W}\left(2 C_{0}+2 m f\right)\right) \otimes H^{0}\left(\mathscr{O}_{W}\left(2 C_{0}+2 m f\right)\right) \stackrel{\beta_{1}}{\longrightarrow} H^{0}\left(\mathscr{O}_{W}\left(4 C_{0}+4 m f\right)\right), \\
H^{0}\left(\mathscr{O}_{W}\left(2 C_{0}+2 m f\right)\right) \otimes H^{0}\left(\mathscr{O}_{W}\left(C_{0}+(m-1) f\right)\right) \\
\stackrel{\beta_{2}}{\longrightarrow} H^{0}\left(\mathscr{O}_{W}\left(3 C_{0}+(3 m-1) f\right)\right), \\
H^{0}\left(\mathscr{O}_{W}\left(2 C_{0}+2 m f\right)\right) \otimes H^{0}\left(\mathscr{O}_{W}((2 m-e-1) f)\right) \\
\stackrel{\beta_{3}}{\longrightarrow} H^{0}\left(\mathscr{O}_{W}\left(2 C_{0}+(4 m-e-1) f\right)\right), \\
H^{0}\left(\mathscr{O}_{W}\left(C_{0}+(m-1) f\right)\right) \otimes H^{0}\left(\mathscr{O}_{W}((2 m-e-1) f)\right) \\
\stackrel{\beta_{4}}{\longrightarrow} H^{0}\left(\mathscr{O}_{W}\left(C_{0}+(3 m-e-2) f\right)\right) .
\end{array}
$$

To prove the surjectivity of $\beta_{1}, \beta_{2}, \beta_{3}$ and $\beta_{4}$ we use Lemma 2.5. Recall that $m \geq$ $e+1$ and $0 \leq e \leq 2$. Then the multiplication map $\beta_{1}$ is surjective by Lemma 2.5 . (a), $\beta_{2}$ by Lemma 2.5 (b), and $\beta_{3}$ and $\beta_{4}$ by Lemma 2.5 (c).

Step 2. To complete the proof, we need to show that the multiplication map (2.6.1) surjects for all $n \geq 2$. This follows from the same argument used for Step 2 of the proof of Theorem 2.4.

Now we focus on the cases in which $X$ is irregular. Under this hypothesis, in the next theorem we find out when $2 K_{X}$ is normally generated.

Theorem 2.7. Let $\varphi: X \longrightarrow W$ be a quadruple Galois canonical cover of Type 3.2. Then $2 K_{X}$ is very ample and $\left|2 K_{X}\right|$ embeds $X$ as a projectively normal variety.

Proof. The proof follows the same arguments as the proof of Theorem 2.6 so we leave the details to the reader.

\section{THE CASE WHERE THE BICANONICAL MORPHISM IS NOT BIRATIONAL ONTO ITS IMAGE}

We have seen in the previous section that if $W$ is smooth and $X$ is regular, then $\varphi_{2}$ embeds $X$ as a projectively normal variety; in particular, $2 K_{X}$ is very ample. We also saw that if $X$ is irregular, then, in one case, $\varphi_{2}$ embeds $X$ as a projectively normal variety (Type 3.2). For the other cases (Types 3.1, 4 and 5) we will prove in this section that $\varphi_{2}$ is not even an embedding; we will compute its degree and we 
will describe $\varphi_{2}(X)$. Thus in this section $X$ will be irregular and, by Theorem 1.3 . $W=\mathbf{F}_{0}$.

Theorem 3.1. Let $\varphi: X \longrightarrow W$ be a quadruple Galois canonical cover of Type 3.1 or 4. Recall that $K_{X}=\varphi^{*} H\left(H=C_{0}+m f ; m=1\right.$ for Type 3.1). Then

(1) the morphism $\varphi_{2}$ is a $2: 1$;

(2) the image of $\varphi_{2}$ is a ruled surface of irregularity $m$. More precisely, $\varphi_{2}(X)=$ $G \times D$, where $G=\mathbf{P}^{1}$ and $D$ is a smooth curve of genus $m$ ( $m=1$ for Type 3.1), embedded by $|2 D+4 m G|$ ( $G$ is embedded as a conic and $D$ is embedded as a projectively normal curve of degree $4 \mathrm{~m}$ ).

Proof. In these cases $W=\mathbf{F}_{0}$. Recall that if $\varphi$ is of Type 3.1, then $\varphi$ factors through a double cover $p_{1}: X^{\prime} \longrightarrow W$, branched along $D_{2} \sim 4 C_{0}$, and a double cover $p_{2}: X \longrightarrow X^{\prime}$ branched along the pullback of a divisor $D_{1} \sim 2 C_{0}+6 f$. If $\varphi$ is of Type 4, then, according to Theorem 1.3, $\varphi$ factors through a double cover $p_{1}: X^{\prime} \longrightarrow W$, branched along $D_{2} \sim(2 m+2) f$, and a double cover $p_{2}: X \longrightarrow X^{\prime}$ branched along the pullback of a divisor $D_{1} \sim 6 C_{0}+2 f$. Thus, if $X$ is of Type 3.1, after applying the involution of $\mathbf{F}_{0}$ we can assume that $D_{1} \sim 6 C_{0}+2 f$ and $D_{2} \sim 4 f$, so Type 3.1 can be considered as a particular case of Type 4 and therefore we will only argue to prove the result for this latter type. Then

$$
\varphi_{*} \mathscr{O}_{X}=\mathscr{O}_{W} \oplus \mathscr{O}_{W}(-(m+1) f) \oplus \mathscr{O}_{W}\left(-3 C_{0}-f\right) \oplus \mathscr{O}_{W}\left(-3 C_{0}-(m+2) f\right)
$$

and the subalgebra of $\varphi_{*} \mathscr{O}_{X}$ that corresponds to $p_{1}$ is

$$
p_{1 *} \mathscr{O}_{X^{\prime}}=\mathscr{O}_{W} \oplus \mathscr{O}_{W}(-(m+1) f) .
$$

Since $K_{X}=\varphi^{*} H$, it follows from the projection formula and from 3.1.1 and 3.1.2 that the global sections of $\mathscr{O}_{X}\left(2 K_{X}\right)$ can be identified with the global sections of $p_{1_{*}} \mathscr{O}_{X^{\prime}} \otimes \mathscr{O}_{W}(2 H)$, so $\varphi_{2}$ factors through $X^{\prime}$. More precisely, $\varphi_{2}=\varphi_{2}^{\prime} \circ p_{2}$, where $\varphi_{2}^{\prime}$ is induced by the complete linear series of $L=p_{1}^{*}\left(\mathscr{O}_{W}(2 H)\right)$.

Now let us study $X^{\prime}$. The structure of $D_{1}$ implies that $X^{\prime}$ is the product of $G=\mathbf{P}^{1}$ and a smooth curve $D$. The curve $D$ is the pullback of $C_{0}$ and has genus $m$. Using the projection formula and the Leray spectral sequence, it follows easily that $H^{1}\left(L \otimes p_{1}^{*}\left(\mathscr{O}_{W}\left(-C_{0}\right)\right)=H^{1}\left(L \otimes p_{1}^{*}\left(\mathscr{O}_{W}(-f)\right)=0\right.\right.$; hence $|L|$ restricts to a complete linear series both on $D$ and on $G$. The restriction of $L$ to $G$ has degree 2 , so $|L|$ maps $G$ onto a smooth conic. On the other hand the restriction of $L$ to $D$ has degree $4 m$, so $|L|$ embeds $D$ as a projectively normal curve of degree $4 m$. Summarizing, $\varphi_{2}$ is $2: 1, \varphi_{2}^{\prime}$ is the embedding of $X^{\prime}$ by the complete linear series $|2 D+4 m G|$ and the image of $\varphi_{2}^{\prime}$ (which is the same as the image of $\varphi_{2}$ ) is the product of a smooth conic and a projectively normal curve of genus $m$ and degree $4 m$.

Theorem 3.2. Let $\varphi: X \longrightarrow W$ be a quadruple Galois canonical cover of Type 5 . Then

(1) the surface $X$ is the product of a smooth curve of genus 2 and a smooth curve of genus $m+1$;

(2.1) if $m=1$ (i.e., $\varphi: X \longrightarrow W$ is of Type 5.1), then the morphism $\varphi_{2}$ is $4: 1$ and its image is $W$;

(2.2) if $m>1$ (i.e., $\varphi: X \longrightarrow W$ is of Type 5.2), then $\varphi_{2}$ is 2:1;

(3.1) if $m=1$, then the image of $\varphi_{2}$ is $\mathbf{F}_{0}$, embedded by $\left|2 C_{0}+2 f\right|$; 
(3.2) if $m>1$, then the image of $\varphi_{2}$ is the product of a smooth, projectively normal curve of genus $m+1$ and degree $4 m$ and a smooth conic.

Proof. Recall (see GP08, Theorem 0.1]) that $\varphi$ is the fiber product of two double covers of $W$, branched along $D_{1} \sim(2 m+4) f$ and $D_{2} \sim 6 C_{0}$, respectively. This implies that $X=G \times D$, where $G$ is a smooth curve of genus 2 (the reduced structure of the pullback of a fiber $f$ by $\varphi$ at the branch locus of $\varphi$ ) and $D$ is a smooth curve of genus $m+1$ (the reduced structure of the pullback of $C_{0}$ by $\varphi$ at the branch locus of $\varphi$ ). This proves (1). Claims (2) and (3) follow easily from (1), since $G$ and $D$ are hyperelliptic.

Theorems 3.1 and 3.2 say in particular that if $X$ is of Type 3.1, 4 or 5 , then $\varphi_{2}$ is not birational. There is a good reason for this to happen, namely, the fact that in these cases $X$ has a pencil of curves of genus 2. In the next remarks we observe that, indeed, if $X$ has a pencil of curves of genus 2, then $\varphi_{2}$ cannot be birational and we describe the pencil of curves of genus 2 if $X$ is of Type 3.1, 4 or 5 .

Remark 3.3. If $X$ has a pencil of genus 2 curves, then this pencil is base-point-free and $\varphi_{2}$ is not birational.

Proof. The pencil of genus 2 curves is base-point-free because of our hypothesis of $K_{X}$ being ample and base-point-free. Then, if $C$ is a general member of the pencil, $\left|2 K_{X}\right|$ restricts to a linear base-point-free subseries of $\left|2 K_{C}\right|$, hence to the complete bicanonical linear series of $C$. Then $\varphi_{2}$ maps $C$ two-to-one onto its image.

Remark 3.4. Surfaces of Types 3.1, 4 and 5 have these pencils of genus 2 curves:

(1) if $X$ is of Type 3.1, $X$ possesses an elliptic pencil of curves of genus 2;

(2) if $X$ is of Type 4, $X$ possesses a genus $m$ pencil of curves of genus 2;

(3) if $X$ is of Type $5, X$ possesses a genus $m+1$ pencil of curves of genus 2 .

Remark 3.5. The existence of pencils of genus 2 curves if $X$ is of Type 3.1, 4 or 5 also follows indirectly from more general results on the classification of the bicanonical maps of surfaces of general type, stated in [Xia90], taking into account what the degree is and what is the image of $\varphi_{2}$ (see Theorems 3.1 and 3.2).

Indeed, if $X$ is of Type 3.1, 4 or 5.2, then Theorems 3.1 and 3.2 say that $\varphi_{2}$ has degree 2. If $X$ did not have a pencil of genus 2 curves, Xia90, Theorem 2] would imply that either $q(X)=0$ or $\varphi_{2}(X)$ is rational. However Theorem 1.3 says that $q(X)>0$. On the other hand, Theorems 3.1 and 3.2 say that the image of $\varphi_{2}$ is a nonrational ruled surface, so $X$ should have a pencil of genus 2 curves.

If $X$ is of Type 5.1, Theorem 1.3 implies that $X$ does not fit in the list of exceptions of [Xia90, Theorem 1]. On the other hand, Theorem 3.2 says that $\varphi_{2}$ has degree 4; hence Xia90, Theorem 1] implies that $X$ should have a pencil of genus 2 curves.

\section{The Bicanonical Morphism When $W$ is Singular}

In this section we prove that if $W$ is singular (and hence, see GP07, $W=$ $S(0,2))$, then $2 K_{X}$ is not very ample. We also see that, in constrast with the case when $W$ is smooth (in which either $\varphi_{2}$ is an embedding with projectively normal image or $\varphi_{2}$ has degree greater than 1) if $W$ is singular, then $\varphi_{2}$ is birational but not an embedding. Precisely, we have the following. 
Theorem 4.1. Let $\varphi: X \longrightarrow W$ be a quadruple Galois canonical cover of $W=$ $S(0,2)$ and let $w$ be the vertex of $W$. Then $\varphi_{2}$ is a birational morphism but $2 K_{X}$ is not very ample because $\varphi_{2}$ fails to be an embedding at $\varphi^{-1}\{w\}$. Precisely,

(1) if $\varphi$ is of Type 6, 7 or 9, then $\left|2 K_{X}\right|$ does not separate directions at the unique point $x \in \varphi^{-1}\{w\}$;

(2) if $\varphi$ is of Type 8 , then $\left|2 K_{X}\right|$ does not separate the two points $x_{1}$ and $x_{2}$ of $\varphi^{-1}\{w\}$, although $\varphi_{2}$ is locally an embedding at both of them.

Moreover, $\varphi_{2}$ is an embedding on the set of smooth points of $X-\varphi^{-1}\{w\}$ and, if $X$ has the mildest possible singularities (see GP07, Corollary 5.1 and Propositions $5.2,5.3$ and 5.4]), then $\varphi_{2}$ is an embedding outside $\varphi^{-1}\{w\}$.

Before we prove Theorem 4.1 we state a couple of lemmas.

Lemma 4.2. Let $S$ be an irreducible, normal surface with only rational singularities, let $\pi: \widetilde{S} \longrightarrow S$ be a proper birational map and let $\mathfrak{m}$ be a maximal ideal sheaf on $S$. Then $\pi_{*}\left(\mathfrak{m}^{n} \mathscr{O}_{\widetilde{S}}\right)=\mathfrak{m}^{n}$.

Proof. The lemma follows from Remark c) of Section 5, Theorem 7.1 and Proposition 6.2 of Lip69.

Lemma 4.3. Let $S$ be a regular surface of general type whose canonical map is a degree $n$ morphism $\psi: S \longrightarrow W$ onto a surface of minimal degree $W$. Let $C \in\left|K_{S}\right|$ be a smooth, irreducible curve. If $C$ is hyperelliptic, then $n=2$.

Proof. Since $K_{S}$ is base-point-free, then $\left.K_{S}\right|_{C}$ is a base-point-free theta-characteristic on $C$. Let $g$ be the genus of $C$. Thus, if $C$ is hyperelliptic, $\left.K_{S}\right|_{C}$ is $\frac{g-1}{2}$ times the $g_{2}^{1}$ of $C$ and $h^{0}\left(\mathscr{O}_{C}\left(\left.K_{S}\right|_{C}\right)\right)=\frac{g+1}{2}$. On the other hand, since $S$ is regular, $h^{0}\left(\mathscr{O}_{C}\left(\left.K_{S}\right|_{C}\right)\right)=p_{g}-1$. Since $W$ is a surface of minimal degree, $g-1=\left.\operatorname{deg} K_{S}\right|_{C}=$ $K_{S}^{2}=n\left(p_{g}-2\right)$; hence $g-1=n\left(p_{g}-2\right)=n\left(\frac{g+1}{2}-1\right)$, so $n=2$.

Proof of Theorem 4.1. We use the notation of (1.3.1). We first prove that if $X$ has the mildest possible singularities, then $\varphi_{2}$ is an embedding outside $\varphi^{-1}\{w\}$. Recall that outside $w, Y$ and $W$ are isomorphic. Also recall that outside $\varphi^{-1}\{w\}, \bar{X}$ and $X$ are isomorphic and $p$ and $\varphi$ are equal. Now let $x_{1}$ and $x_{2}$ be two distinct points of $X-\varphi^{-1}\{w\}$. If $\varphi\left(x_{1}\right) \neq \varphi\left(x_{2}\right)$, then $\varphi_{2}\left(x_{1}\right) \neq \varphi_{2}\left(x_{2}\right)$, since $\varphi_{2}(X)$ can be projected to a 2-Veronese reembedding of $W$. Thus, let us assume $\varphi\left(x_{1}\right)=\varphi\left(x_{2}\right)$ and let us call $y$ the inverse image of $\varphi\left(x_{1}\right)=\varphi\left(x_{2}\right)$ by $q$. Consider the linear system $\left|C_{0}+2 f\right|$ in $Y$. Since it is base-point-free and big, there exists a smooth connected curve in $\left|C_{0}+2 f\right|$, not meeting $C_{0}$, passing through $y$ and meeting the branch locus of $p$ in such a way that its pullback $C$ by $p$ is smooth and irreducible. Then $\bar{q}(C)$ is isomorphic to $C$ and belongs to $\left|K_{X}\right|$, is smooth and irreducible and passes through $x_{1}$ and $x_{2}$. Since $\varphi$ has degree $4, C$ is not hyperelliptic by Lemma 4.3. Since $X$ is regular, $\left|2 K_{X}\right|$ restricts to the complete canonical series of $C$; thus $\left|2 K_{X}\right|$ embeds $C$ and therefore separates $x_{1}$ and $x_{2}$. A similar argument proves that in general $\varphi_{2}$ separates smooth points of $X-\varphi^{-1}\{w\}$. Moreover, this argument can be adapted to show that $\varphi_{2}$ separates directions in the set of smooth points of $X-\varphi^{-1}\{w\}$.

Now we see what happens at $\varphi^{-1}\{w\}$. First we treat the case in which $\varphi^{-1}\{w\}$ consists of only one point $x$ (Types 6,7 and 9 ). Let $\mathscr{O}_{X, x}$ be the local ring at $x$, let $\mathfrak{m}_{x}$ be the maximal ideal of $x$ and let

$$
Z=\operatorname{Spec} \mathscr{O}_{X, x} / \mathfrak{m}_{x}^{2}
$$


be the second infinitesimal neighbourhood of $x$. We want to prove that $\left|2 K_{X}\right|$ does not separate directions at $x$. This is equivalent to proving that

$$
H^{0}\left(\mathscr{O}_{X}\left(2 K_{X}\right)\right) \longrightarrow H^{0}\left(\left.\mathscr{O}_{X}\left(2 K_{X}\right)\right|_{Z}\right)
$$

is not surjective. Since, by Kodaira vanishing, $H^{1}\left(\mathscr{O}_{X}\left(2 K_{X}\right)\right)=0$, the latter is equivalent to the nonvanishing of $H^{1}\left(\mathscr{O}_{X}\left(2 K_{X}\right) \otimes \mathfrak{m}_{x}^{2}\right)$. To study this cohomology group we will use Lemma4.2. Thus we will compute $H^{1}\left(\mathscr{O}_{X}\left(2 K_{X}\right) \otimes \mathfrak{m}_{x}^{2}\right)$ by arguing on $\bar{X}$ and with $L=\bar{q}^{*} \mathscr{O}_{X}\left(K_{X}\right)=p^{*}\left(\mathscr{O}_{W}\left(C_{0}+2 f\right)\right)$. We denote $F=p^{-1} C_{0}$.

Now we argue for each Type 6,7 and 9 . We start with Type 9 . In this case (see [GP07, Corollary 5.1]) the morphism $\bar{X} \stackrel{\bar{q}}{\longrightarrow} X$ is the contraction of the smooth line $F$. The line $F$ consists of smooth points of $\bar{X}$ and an $A_{1}$ singularity $\bar{x}$. Also recall that the self-intersection of $F$ is $F^{2}=-\frac{1}{2}$ and that $p^{*} C_{0}=4 F$. Thus $\bar{q}$ factorizes as a composition of blowing ups and blowing downs in the following way: Let $g: \widehat{X} \longrightarrow \bar{X}$ be the blowing up of $\bar{X}$ at $\bar{x}$. Then the exceptional divisor $F_{1}$ of $g$ has $F_{1}^{2}=-2$, and the strict transform of $F$ is a line $F_{2}$ with $F_{2}^{2}=-1$. Then we obtain $X$ from $\widehat{X}$ by contracting first $F_{2}$ and then $F_{1}$, so $\widehat{X}$ is obtained from $X$ by performing two consecutive blowing ups, $g_{1}: X^{\prime} \longrightarrow X$ and $g_{2}: \widehat{X} \longrightarrow X^{\prime}$, the first one centered at $x$. Let us call $M=g_{2}^{*}\left(g_{1}^{*} K_{X}\right)$ and recall that $L=\bar{q}^{*} \mathscr{O}_{X}\left(K_{X}\right)=p^{*}\left(\mathscr{O}_{W}\left(C_{0}+2 f\right)\right)$. Also notice that $\mathscr{O}_{\widehat{X}}(M)=g^{*} L$. Then, local computations and Lemma 4.2 yield

$$
\left(g_{2} \circ g_{1}\right)_{*} \mathscr{O}_{\widehat{X}}\left(-2 F_{1}-2 F_{2}\right)=\mathfrak{m}_{x}^{2} .
$$

Then, by the projection formula,

$$
\left(g_{2} \circ g_{1}\right)_{*}\left(\mathscr{O}_{\widehat{X}}(2 M) \otimes \mathscr{O}_{\widehat{X}}\left(-2 F_{1}-2 F_{2}\right)\right)=\mathscr{O}_{X}\left(2 K_{X}\right) \otimes \mathfrak{m}_{x}^{2}
$$

and by the Leray Spectral Sequence,

$H^{1}\left(\left(g_{2} \circ g_{1}\right)_{*}\left(\mathscr{O}_{\widehat{X}}(2 M) \otimes \mathscr{O}_{\widehat{X}}\left(-2 F_{1}-2 F_{2}\right)\right)\right)=H^{1}\left(\mathscr{O}_{\widehat{X}}(2 M) \otimes \mathscr{O}_{\widehat{X}}\left(-2 F_{1}-2 F_{2}\right)\right)$.

Local computation shows that

$$
\begin{aligned}
& \mathscr{O}_{\bar{X}}(-2 F) \cdot \mathscr{O}_{\widehat{X}}=\mathscr{O}_{\widehat{X}}\left(-F_{1}-2 F_{2}\right) \text { and that } \\
& \left(\mathscr{O}_{\bar{X}}(-2 F) \otimes m_{\bar{x}}\right) \cdot \mathscr{O}_{\widehat{X}}=\mathscr{O}_{\widehat{X}}\left(-2 F_{1}-2 F_{2}\right),
\end{aligned}
$$

where $\mathfrak{m}_{\bar{x}}$ is the maximal ideal corresponding to $\bar{x}$. Then Remarks c) and e) of Section 5 and Proposition 6.2 of Lip69 and Lemma 4.2 show that

$$
\begin{gathered}
g_{*} \mathscr{O}_{\widehat{X}}\left(-F_{1}-2 F_{2}\right)=\mathscr{O}_{\bar{X}}(-2 F) \text { and } \\
g_{*} \mathscr{O}_{\widehat{X}}\left(-2 F_{1}-2 F_{2}\right)=\mathscr{O}_{\bar{X}}(-2 F) \otimes \mathfrak{m}_{\bar{x}},
\end{gathered}
$$

where $\mathfrak{m}_{\bar{x}}$ is the maximal ideal corresponding to $\bar{x}$. Thus we consider the exact sequence

$$
\begin{aligned}
0 \longrightarrow \mathscr{O}_{\widehat{X}}(2 M) \otimes \mathscr{O}_{\widehat{X}}\left(-2 F_{1}-2 F_{2}\right) \longrightarrow & \mathscr{O}_{\widehat{X}}(2 M) \otimes \mathscr{O}_{\widehat{X}}\left(-F_{1}-2 F_{2}\right) \\
& \longrightarrow \mathscr{O}_{\widehat{X}}(2 M) \otimes \mathscr{O}_{F_{1}}\left(-F_{1}-2 F_{2}\right) \longrightarrow 0
\end{aligned}
$$

and push it down to $\bar{X}$ to obtain

$$
0 \longrightarrow g_{*}\left(\mathscr{O}_{\widehat{X}}(2 M) \otimes \mathscr{O}_{\widehat{X}}\left(-2 F_{1}-2 F_{2}\right)\right) \longrightarrow 2 L \otimes \mathscr{O}_{\bar{X}}(-2 F) \longrightarrow \mathbf{k}_{\bar{x}} \longrightarrow 0
$$

where $\mathbf{k}_{\bar{x}}$ is the skyscraper sheaf on $\bar{x}$ of dimension 1 obtained by restricting $\mathscr{O}_{\widehat{X}}(2 M) \otimes \mathscr{O}_{F_{1}}\left(-F_{1}-2 F_{2}\right)$ to $\bar{x}$. The above sequence is exact because $R^{1} g_{*}\left(\mathscr{O}_{\widehat{X}}(2 M)\right.$ $\left.\otimes \mathscr{O}_{\widehat{X}}\left(-2 F_{1}-2 F_{2}\right)\right)$ vanishes. Again by the Leray Spectral Sequence, $H^{1}\left(\mathscr{O}_{\widehat{X}}(2 M) \otimes\right.$ 
$\left.\mathscr{O}_{\widehat{X}}\left(-2 F_{1}-2 F_{2}\right)\right)=H^{1}\left(g_{*}\left(\mathscr{O}_{\widehat{X}}(2 M) \otimes \mathscr{O}_{\widehat{X}}\left(-2 F_{1}-2 F_{2}\right)\right)\right)$, so we take cohomology in the above sequence (4.3.1). So, if we see that $H^{1}\left(2 L \otimes \mathscr{O}_{\bar{X}}(-2 F)\right) \neq 0$, then $H^{1}\left(\mathscr{O}_{\widehat{X}}(2 M) \otimes \mathscr{O}_{\widehat{X}}\left(-2 F_{1}-2 F_{2}\right)\right) \neq 0$ and we are done. Then, to see that $H^{1}\left(2 L \otimes \mathscr{O}_{\bar{X}}(-2 F)\right) \neq 0$, we argue like this. From [GP07, Corollary 5.1], $K_{\bar{X}}=$ $\bar{q}^{*} K_{X}+2 F$; hence

$$
\begin{aligned}
H^{1}\left(2 L \otimes \mathscr{O}_{\bar{X}}(-2 F)\right) & =H^{1}\left(\mathscr{O}_{\bar{X}}\left(K_{\bar{X}}-4 F\right) \otimes L\right) \\
& =H^{1}\left(\mathscr{O}_{\bar{X}}\left(K_{\bar{X}}+p^{*}(2 f)\right)\right)=H^{1}\left(\mathscr{O}_{\bar{X}}\left(p^{*}(-2 f)\right)\right)^{*} .
\end{aligned}
$$

Now by [GP07, Corollary 4.3],

$$
p_{*} \mathscr{O}_{\bar{X}}=\mathscr{O}_{Y} \oplus \mathscr{O}_{Y}\left(-2 C_{0}-3 f\right) \oplus \mathscr{O}_{Y}\left(-2 C_{0}-3 f\right) \oplus \mathscr{O}_{Y}\left(-3 C_{0}-6 f\right),
$$

so

$p_{*} p^{*}\left(\mathscr{O}_{Y}(-2 f)\right)=\mathscr{O}_{Y}(-2 f) \oplus \mathscr{O}_{Y}\left(-2 C_{0}-5 f\right) \oplus \mathscr{O}_{Y}\left(-2 C_{0}-5 f\right) \oplus \mathscr{O}_{Y}\left(-3 C_{0}-8 f\right)$.

Then, by (4.3.3) and the Leray Spectral Sequence, $\left.h^{1}\left(p^{*} \mathscr{O}_{Y}(-2 f)\right)\right)=h^{1}\left(\mathscr{O}_{Y}(-2 f)\right)$ $=1$.

Now we deal with Type 7 . In this case $\bar{q}$ is the blowup of $X$ at $x$ and a partial desingularization of $X$ at $x$ (recall that $x$ is a $D_{4}$ singularity). Also recall that the exceptional divisor of $\bar{q}$ is a line $F$ and in this case we have, as in Type 9 , that $p^{*} C_{0}=$ $4 F$. The points of $F$ are smooth except 3 points which are $A_{1}$ singularities and $F^{2}=-1 / 2$. The local equation of $X$ at $x$ is $z^{2} t-t^{3}-u^{2}=0$ and a local computation of the blowing up at $x$ shows that $\mathfrak{m}_{x} \mathscr{O}_{\bar{X}}=\mathscr{O}_{\bar{X}}(-2 F)$ and $\mathfrak{m}_{x}^{2} \mathscr{O}_{\bar{X}}=\mathscr{O}_{\bar{X}}(-4 F)$. Now recall that we want to prove the nonvanishing of $H^{1}\left(\mathscr{O}_{X}\left(2 K_{X}\right) \otimes \mathfrak{m}_{x}^{2}\right)$. Recall that $L=\bar{q}^{*} \mathscr{O}_{X}\left(K_{X}\right)=p^{*}\left(\mathscr{O}_{Y}\left(C_{0}+2 f\right)\right)$. By Lemma 4.2, $\bar{q}_{*}\left(\mathscr{O}_{\bar{X}}(-4 F)\right)=\mathfrak{m}_{x}^{2}$; hence, by the projection formula and the Leray spectral sequence,

$$
H^{1}\left(\mathscr{O}_{X}\left(2 K_{X}\right) \otimes \mathfrak{m}_{x}^{2}\right)=H^{1}\left(p^{*}\left(\mathscr{O}_{Y}\left(C_{0}+4 f\right)\right)\right) .
$$

Also recall that in Type 7 (see GP07, Corollary 4.3]),

$$
p_{*} \mathscr{O}_{\bar{X}}=\mathscr{O}_{Y} \oplus \mathscr{O}_{Y}\left(-C_{0}-3 f\right) \oplus \mathscr{O}_{Y}\left(-2 C_{0}-3 f\right) \oplus \mathscr{O}_{Y}\left(-3 C_{0}-6 f\right) .
$$

Thus, by the projection formula, the Leray Spectral Sequence and Serre duality, $h^{1}\left(p^{*}\left(\mathscr{O}_{Y}\left(C_{0}+4 f\right)\right)\right)=h^{1}\left(\mathscr{O}_{Y}\left(-2 C_{0}-2 f\right)\right)=h^{1}\left(\mathscr{O}_{Y}(-2 f)\right)=1$, so $H^{1}\left(\mathscr{O}_{X}\left(2 K_{X}\right) \otimes\right.$ $\left.\mathfrak{m}_{x}^{2}\right) \neq 0$, as wanted.

Now we deal with Type 6 . In this case, $p^{*} C_{0}=2 F$ and $F^{2}=-2$, so $x$ is an $A_{1}$ singularity, and $\bar{q}$ is the blowup of $X$ at $x$, which desingularizes $X$. Then $\mathfrak{m}_{x}^{2} \mathscr{O}_{\bar{X}}=$ $\mathscr{O}_{\bar{X}}(-2 F)$, so arguing as in the cases above and using Lemma 4.2, the projection formula and the Leray Spectral Sequence we have that $H^{1}\left(2 L \otimes \mathscr{O}_{\bar{X}}(-2 F)\right)=$ $H^{1}\left(\mathscr{O}_{X}\left(2 K_{X}\right) \otimes \mathfrak{m}_{x}^{2}\right)$. Recall that $L=\bar{q}^{*} \mathscr{O}_{X}\left(K_{X}\right)=p^{*}\left(\mathscr{O}_{Y}\left(C_{0}+2 f\right)\right)$, so $H^{1}(2 L \otimes$ $\left.\mathscr{O}_{\bar{X}}(-2 F)\right)=H^{1}\left(p^{*}\left(\mathscr{O}_{Y}\left(C_{0}+4 f\right)\right)\right)$. Since $p_{*} \mathscr{O}_{\bar{X}}$ splits as (4.3.4) (see GP07, Corollary 4.3]), we obtain that $h^{1}\left(\mathscr{O}_{Y}(-2 f)\right)=1$ as in Type 7 .

Finally we study the case in which $\varphi^{-1}\{w\}$ consists of two points, $x_{1}$ and $x_{2}$. This is a quadruple Galois canonical cover of Type 8. We prove first that $\left|2 K_{X}\right|$ does not separate $x_{1}$ and $x_{2}$. Recall (see GP07, Corollary 5.1]) that $x_{1}$ and $x_{2}$ are smooth points and $\bar{q}$ is the blowing up of $X$ at $x_{1}$ and $x_{2}$, so $\bar{q}_{*} \mathscr{O}_{\bar{X}}\left(-F_{1}-F_{2}\right)=\mathfrak{m}_{x_{1}} \otimes \mathfrak{m}_{x_{2}}$, where $F_{1}$ and $F_{2}$ are the exceptional divisors of $\bar{q}$, which are -1 -lines. Let $f$ be a general fiber of the ruled surface $Y$ and let $\bar{f}$ be the pullback to $\bar{X}$ of $f$ by $p$. Then $\bar{f}$ is a smooth, connected curve of genus 4 , meeting $F_{1}$ (respectively $F_{2}$ ) at one point $\bar{x}_{1}$ (respectively $\bar{x}_{2}$ ) transversally (see the proof of GP07, Theorem 4.1]). Recall also that $\bar{q}^{*} \mathscr{O}_{X}\left(K_{X}\right)=L=p^{*}\left(\mathscr{O}_{Y}\left(C_{0}+2 f\right)\right)$ and that the morphism is 
induced on $\bar{X}$ by $|2 L|$ factors through $\varphi_{2}$. Then, if the restriction of $|2 L|$ to $\bar{f}$ does not separate $\bar{x}_{1}$ and $\bar{x}_{2}$, then $\varphi_{2}\left(x_{1}\right)=\varphi_{2}\left(x_{2}\right)$. Note that the degree of $\left.2 L\right|_{\bar{f}}$ is 8 . Also recall that $K_{\bar{X}}=p^{*}\left(C_{0}+2 f\right)+F_{1}+F_{2}$. Then, by the adjunction formula, $\left.2 L\right|_{\bar{f}}$ is the canonical of $\bar{f}$ plus the degree 2 , effective divisor $\left.\left(F_{1}+F_{2}\right)\right|_{\bar{f}}=\bar{x}_{1}+\bar{x}_{2}$. Therefore the restriction of $|2 L|$ to $\bar{f}$ does not separate $\bar{x}_{1}$ and $\bar{x}_{2}$.

Now we show that $\varphi_{2}$ is a local embedding at both $x_{1}$ and $x_{2}$. Let

$$
Z^{\prime}=\operatorname{Spec}\left(\mathscr{O}_{X, x_{1}} / \mathfrak{m}_{x_{1}}^{2} \oplus \mathscr{O}_{X, x_{2}} / \mathfrak{m}_{x_{2}}^{2}\right) \text {. }
$$

We will show that the cokernel of the homomorphism

$$
H^{0}\left(\mathscr{O}_{Y}\left(2 K_{X}\right)\right) \longrightarrow H^{0}\left(\left.\mathscr{O}_{Y}\left(2 K_{X}\right)\right|_{Z^{\prime}}\right)
$$

has dimension 1. Since $\varphi_{2}\left(x_{1}\right)=\varphi_{2}\left(x_{2}\right)$, in that case it would follow that $\varphi_{2}$ is a local embedding at both $x_{1}$ and $x_{2}$, so we would be done. Thus, we show now that the cokernel of 4.3.5) has dimension 1. Since $H^{1}\left(\mathscr{O}_{X}\left(2 K_{X}\right)\right)=0$, this is equivalent to showing that $h^{1}\left(\mathscr{O}_{X}\left(2 K_{X}\right) \otimes \mathfrak{m}_{x_{1}}^{2} \otimes \mathfrak{m}_{x_{2}}^{2}\right)=1$. For the latter, since $\bar{q}_{*} \mathscr{O}_{\bar{X}}\left(-2 F_{1}-2 F_{2}\right)=\mathfrak{m}_{x_{1}}^{2} \otimes \mathfrak{m}_{x_{2}}^{2}$, from the projection formula and the Leray Spectral Sequence it follows that

$$
H^{1}\left(\mathscr{O}_{X}\left(2 K_{X}\right) \otimes \mathfrak{m}_{x_{1}}^{2} \otimes \mathfrak{m}_{x_{2}}^{2}\right)=H^{1}\left(2 L \otimes \mathscr{O}_{\bar{X}}\left(-2 F_{1}-2 F_{2}\right)\right) .
$$

The latter cohomology group is equal to $H^{1}\left(p^{*}\left(\mathscr{O}_{Y}\left(C_{0}+4 f\right)\right)\right)$. The splitting of $p_{*} \mathscr{O}_{\bar{X}}$ is [4.3.2) (see GP07, Corollary 4.3]), so the projection formula, the Leray Spectral Sequence and Serre duality yield

$$
\begin{aligned}
h^{1}\left(p^{*}\left(\mathscr{O}_{Y}\left(C_{0}+4 f\right)\right)\right) & =h^{1}\left(\mathscr{O}_{Y}\left(-2 C_{0}-4 f\right)\right)=h^{1}\left(\mathscr{O}_{Y}(-2 f)\right)=1, \\
\text { so } h^{1}\left(\mathscr{O}_{X}\left(2 K_{X}\right) \otimes \mathfrak{m}_{x_{1}}^{2} \otimes \mathfrak{m}_{x_{2}}^{2}\right) & =1 .
\end{aligned}
$$

\section{Ring Generators of the CANONiCAL Ring OF QUADRUPLE CANONICAL COVERS}

In this section we study the generators of the canonical ring of a quadruple Galois canonical cover $X$ of minimal degree. Precisely we will find the degrees of the minimal generators of the canonical ring of $X$, and the number of generators in each degree. The first result of this section is a general result that gives a beautiful formula for the number of generators in degree 2 of the canonical ring of canonical covers, of arbitrary degree and irregularity, of surfaces of minimal degree. This result recovers part of [GP03, Theorem 2.1], which gives the degrees and number of generators of the canonical ring of a regular surface $S$ of general type with at worst canonical singularities, that is, a canonical cover of a surface of minimal degree. One of the consequences of GP03, Theorem 2.1] is that the number of extra generators needed in degree 2 depends only on the geometric genus $p_{g}(S)$ of $S$ and on the degree $n$ of $\Psi$; precisely [GP03, Theorem 2.1] tells us that this number is $(n-2)\left(p_{g}(S)-2\right)$. This formula is generalized to canonical covers of arbitrary irregularity of surfaces of minimal degree in the following:

Theorem 5.1. Let $S$ be a surface of general type, normal with at worst canonical singularities and such that its canonical bundle is base-point-free. Let $\Psi$ be the canonical morphism of $S$ and let $n$ be the degree of $\Psi$. Assume that the image of $\Psi$ is a surface $W$ of minimal degree. Then, the degree 2 part of the canonical ring $R(S)$ of $S$ is generated by the elements of degree 1 of $R(S)$ and by $(n-2)\left(p_{g}(S)-2\right)-q(S)$ linearly independent elements of degree 2 . 
Proof. The part $R_{2}$ of degree 2 of $R(S)$ is $H^{0}\left(\mathscr{O}_{S}\left(2 K_{S}\right)\right)$. Then the number of linearly independent elements of degree 2 which, in addition to the elements of degree 1 , are needed to generate $R_{2}$ is the dimension of the cokernel of the multiplication map of global sections on $S$,

$$
H^{0}\left(\mathscr{O}_{S}\left(K_{S}\right)\right) \otimes H^{0}\left(\mathscr{O}_{S}\left(K_{S}\right)\right) \longrightarrow H^{0}\left(\mathscr{O}_{S}\left(2 K_{S}\right)\right)
$$

which is equal to the dimension of the cokernel of the multiplication map of global sections on $W$,

$$
H^{0}\left(\Psi_{*} \mathscr{O}_{S}\left(K_{S}\right)\right) \otimes H^{0}\left(\Psi_{*} \mathscr{O}_{S}\left(K_{S}\right)\right) \stackrel{\gamma^{\prime}}{\longrightarrow} H^{0}\left(\Psi_{*} \mathscr{O}_{S}\left(2 K_{S}\right)\right) .
$$

We have an exact sequence

$$
0 \longrightarrow \mathscr{O}_{W} \stackrel{i}{\longrightarrow} \Psi_{*} \mathscr{O}_{S} \longrightarrow \mathscr{F} \longrightarrow 0
$$

of sheaves on $W$, where $\mathscr{F}$ is simply the cokernel of $i$. Let $H$ be the hyperplane section of $W$. Then $K_{S}=\Psi^{*} H$ and $H^{0}\left(\mathscr{O}_{S}\left(K_{S}\right)\right)=H^{0}\left(\Psi_{*} \mathscr{O}_{S}\left(K_{S}\right)\right)=H^{0}\left(\mathscr{O}_{W}(H)\right)$, the last equality being induced by $i$. On the other hand,

$$
H^{0}\left(\mathscr{O}_{W}(H)\right) \otimes H^{0}\left(\mathscr{O}_{W}(H)\right) \longrightarrow H^{0}\left(\mathscr{O}_{W}(2 H)\right)
$$

surjects, because $W$ is projectively normal. Then the image of $\gamma^{\prime}$ is $H^{0}\left(\mathscr{O}_{W}(2 H)\right)$, which by (5.1.1) is a subspace of $H^{0}\left(\Psi_{*} \mathscr{O}_{S}\left(2 K_{S}\right)\right)$. Therefore the dimension of the cokernel of $\gamma^{\prime}$ is $h^{0}\left(\Psi_{*} \mathscr{O}_{S}\left(2 K_{S}\right)\right)-h^{0}\left(\mathscr{O}_{W}(2 H)\right)=h^{0}\left(\mathscr{O}_{S}\left(2 K_{S}\right)\right)-h^{0}\left(\mathscr{O}_{W}(2 H)\right)$. By Riemann-Roch, Serre duality and the Kawamata-Viehweg vanishing theorem, $h^{0}\left(\mathscr{O}_{S}\left(2 K_{S}\right)\right)=K_{S}^{2}+p_{g}(S)-q(S)+1$. Now let $C$ be a smooth curve in the linear system $|H|$, not meeting the singular locus of $W$. Then $H^{0}\left(\mathscr{O}_{W}(2 H)\right)$ fits in the exact sequence

$$
0 \longrightarrow H^{0}\left(\mathscr{O}_{W}(H)\right) \longrightarrow H^{0}\left(\mathscr{O}_{W}(2 H)\right) \longrightarrow H^{0}\left(\mathscr{O}_{C}\left(\left.2 H\right|_{C}\right)\right) \longrightarrow 0
$$

since $H^{1}\left(\mathscr{O}_{W}(H)\right)=0$, which follows from the fact that $W$ is a regular surface. Since $C=\mathbf{P}^{1}, h^{0}\left(\mathscr{O}_{C}\left(\left.2 H\right|_{C}\right)\right)=2 H^{2}+1$, so $h^{0}\left(\mathscr{O}_{W}(2 H)\right)=p_{g}(S)+2 H^{2}+1$. Now we have that $K_{S}^{2}=n H^{2}$ and, since $W$ is a surface of minimal degree, $H^{2}=p_{g}(S)-2$. All this yields $h^{0}\left(\mathscr{O}_{S}\left(2 K_{S}\right)\right)-h^{0}\left(\mathscr{O}_{W}(2 H)\right)=(n-2)\left(p_{g}(S)-2\right)-q(S)$.

The result [GP03, Theorem 2.1] tells us among other things that, unless $n=2$, the canonical ring of $S$ is generated in degree less than or equal to 3. Theorem 5.1 gives a nice, uniform formula for the number of generators in degree 2, depending only on the geometric and arithmetic genus of $S$. Thus a natural question to ask is whether there exists a uniform formula or pattern for the number of generators in degree 3, similar to the one for generators of degree 2. Indeed, [GP03, Theorem 2.1] gives us such a formula when $S$ is regular (precisely, the number of extra generators in degree 3 is $p_{g}(S)-3$ if $S$ is regular and $n \neq 2$ ). However, irregular quadruple Galois canonical covers of Types 3.1, 4 and 5 show that this formula cannot be generalized for an arbitrary $S$, as one can see in Theorem 5.2 below:

Theorem 5.2. Let $\varphi: X \longrightarrow W$ be a quadruple Galois canonical cover of a surface of minimal degree. Then the canonical ring of $X$ is generated in degree less than or equal to 3. Precisely, the canonical ring of $X$ is generated by its part of degree 1 , by $2 p_{g}(X)-4-q(X)$ extra generators in degree 2 , and by $\delta(X)$ extra generators in degree 3 , where

a) $\delta(X)=p_{g}(X)-3$ if $X$ is of Type 1, 2, 3.2, 6, 7, 8 or 9;

b) $\delta(X)=4$ if $X$ is of Type 3.1; 
c) $\delta(X)=5 m-1$ if $X$ is of Type 4;

d) $\delta(X)=9$ if $X$ is of Type 5.1;

e) $\delta(X)=5 m$ if $X$ is of Type 5.2.

Proof. Let $R$ be the canonical ring of $X$ and $R_{n}=H^{0}\left(\mathscr{O}_{X}\left(n K_{X}\right)\right)$ its part of degree $n$. If $X$ is regular (i.e., if $X$ is of Type $1,2,6,7,8$ or 9 ) the result follows from GP03, Theorem 2.1]. The part of the result regarding $R_{2}$, for any surface $X$, follows from Theorem 5.1 .

Then it only remains to prove that $R$ is generated in degree less than or equal to 3 and to find the number of extra generators of degree 3 if $X$ is of Type 3,4 or 5 . For this we will study the multiplication maps of global sections of line bundles on $X$,

$$
H^{0}\left(\mathscr{O}_{X}\left(K_{X}\right)\right) \otimes H^{0}\left(\mathscr{O}_{X}\left(n K_{X}\right)\right) \stackrel{\gamma_{n}}{\longrightarrow} H^{0}\left(\mathscr{O}_{X}\left((n+1) K_{X}\right)\right),
$$

when $n \geq 2$. The dimension of the cokernel of $\gamma_{2}$ is the number of linearly independent elements of degree 3 which are required, together with the elements of $R_{1}$ and $R_{2}$, to generate $R_{3}$. On the other hand, if $\gamma_{n}$ is surjective for all $n \geq 3$, then $R$ is generated by its elements of degree less than or equal to 3 . Now, to study the maps $\gamma_{n}$, we look at the $\mathscr{O}_{W}$-algebra structure of $\varphi_{*} \mathscr{O}_{X}$ and use Lemma 2.1 and arguments similar to those used in Section 2, Recall that, by Theorem 1.3 $W=\mathbf{F}_{0}$ and that the splitting (2.0.2) of $\varphi_{*} \mathscr{O}_{X}$ is, for $X$ of the following types:

Type $3, \quad \mathscr{O}_{W} \oplus \mathscr{O}_{W}\left(-C_{0}-(m+2) f\right) \oplus \mathscr{O}_{W}\left(-2 C_{0}\right) \oplus \mathscr{O}_{W}\left(-3 C_{0}-(m+2) f\right)$;

Type 4, $\mathscr{O}_{W} \oplus \mathscr{O}_{W}(-(m+1) f) \oplus \mathscr{O}_{W}\left(-3 C_{0}-f\right) \oplus \mathscr{O}_{W}\left(-3 C_{0}-(m+2) f\right)$;

Type 5, $\quad \mathscr{O}_{W} \oplus \mathscr{O}_{W}(-(m+2) f) \oplus \mathscr{O}_{W}\left(-3 C_{0}\right) \oplus \mathscr{O}_{W}\left(-3 C_{0}-(m+2) f\right)$.

We look first at $\gamma_{2}$. Recall that $H^{0}\left(\varphi_{*} \mathscr{O}_{X}\left(K_{X}\right)\right)$ has in all cases one nonzero summand, namely, $H^{0}\left(\mathscr{O}_{W}\left(C_{0}+m f\right)\right)$. First we consider $X$ of Type 3.1. It follows from (5.2.1) that the splitting (2.1.1) of $H^{0}\left(\varphi_{*} \mathscr{O}_{X}\left(2 K_{X}\right)\right)$ has two nonzero summands, namely $H^{0}\left(\mathscr{O}_{W}\left(2 C_{0}+2 f\right)\right)$ and $H^{0}\left(\mathscr{O}_{W}(2 f)\right)$, corresponding to $\mathscr{O}_{W}$ and $L_{2}^{*}$ of (2.0.2). On the other hand, the splitting (2.1.1) of $H^{0}\left(\varphi_{*} \mathscr{O}_{X}\left(3 K_{X}\right)\right)$ has four nonzero summands, namely, $H^{0}\left(\mathscr{O}_{W}\left(3 C_{0}+3 f\right)\right), H^{0}\left(\mathscr{O}_{W}\left(2 C_{0}\right)\right), H^{0}\left(\mathscr{O}_{W}\left(C_{0}+3 f\right)\right)$ and $H^{0}\left(\mathscr{O}_{W}\right)$. Then Lemma 2.1 implies that $\gamma_{2}$ is not surjective. More precisely, since $\gamma_{2}$ splits in several summands according to the algebra structure of $\varphi_{*} \mathscr{O}_{X}$, described in GP08, Remark 3.1], it follows that the image of $\gamma_{2}$ is contained in $H^{0}\left(\mathscr{O}_{W}\left(3 C_{0}+3 f\right)\right) \oplus H^{0}\left(\mathscr{O}_{W}\left(C_{0}+3 f\right)\right)$. Moreover, $\gamma_{2}$ surjects onto $H^{0}\left(\mathscr{O}_{W}\left(3 C_{0}+3 f\right)\right) \oplus H^{0}\left(\mathscr{O}_{W}\left(C_{0}+3 f\right)\right)$ if the following multiplication maps on $W$,

$$
\begin{gathered}
H^{0}\left(\mathscr{O}_{W}\left(C_{0}+f\right)\right) \otimes H^{0}\left(\mathscr{O}_{W}\left(2 C_{0}+2 f\right)\right) \longrightarrow H^{0}\left(\mathscr{O}_{W}\left(3 C_{0}+3 f\right)\right), \\
H^{0}\left(\mathscr{O}_{W}\left(C_{0}+f\right)\right) \otimes H^{0}\left(\mathscr{O}_{W}(2 f)\right) \longrightarrow H^{0}\left(\mathscr{O}_{W}\left(C_{0}+3 f\right)\right),
\end{gathered}
$$

surject. This follows from Lemma 2.5 (e). Then the dimension of the cokernel of $\gamma_{2}$ is $h^{0}\left(\mathscr{O}_{W}\left(2 C_{0}\right)\right)+h^{0}\left(\mathscr{O}_{W}\right)=4$.

Arguing similarly, in all other Types 3.2, 4 and 5 we see that, from (5.2.1), the splitting (2.1.1) of $H^{0}\left(\varphi_{*} \mathscr{O}_{X}\left(2 K_{X}\right)\right)$ has these nonzero summands:

$H^{0}\left(\mathscr{O}_{W}\left(2 C_{0}+2 m f\right)\right) \oplus H^{0}\left(\mathscr{O}_{W}\left(C_{0}+(m-2) f\right)\right) \oplus H^{0}\left(\mathscr{O}_{W}(2 m f)\right)$ if $X$ is of Type 3.2 (recall that in such a case, $m \geq 2$ );

$H^{0}\left(\mathscr{O}_{W}\left(2 C_{0}+2 m f\right)\right) \oplus H^{0}\left(\mathscr{O}_{W}\left(2 C_{0}+(m-1) f\right)\right)$ if $X$ is of Type 4;

$H^{0}\left(\mathscr{O}_{W}\left(2 C_{0}+2 f\right)\right)$ if $X$ is of Type 5.1; and

$H^{0}\left(\mathscr{O}_{W}\left(2 C_{0}+2 m f\right)\right) \oplus H^{0}\left(\mathscr{O}_{W}\left(2 C_{0}+(m-2) f\right)\right)$ if $X$ is of Type 5.2 (recall

that in such a case, $m \geq 2)$. 
On the other hand, since $\gamma_{2}$ splits in several summands according to the algebra structure of $\varphi_{*} \mathscr{O}_{X}$, described in [GP08, Remark 3.1], it follows that the image of $\gamma_{2}$ is contained in the subspace $B$ of $H^{0}\left(\varphi_{*} \mathscr{O}_{X}\left(3 K_{X}\right)\right)$, where $B$ is

$$
\begin{aligned}
& H^{0}\left(\mathscr{O}_{W}\left(3 C_{0}+3 m f\right)\right) \oplus H^{0}\left(\mathscr{O}_{W}\left(2 C_{0}+(2 m-2) f\right)\right) \oplus H^{0}\left(\mathscr{O}_{W}\left(C_{0}+3 m f\right)\right) \\
& \text { if } X \text { is of Type } 3.2 ; \\
& H^{0}\left(\mathscr{O}_{W}\left(3 C_{0}+3 m f\right)\right) \oplus H^{0}\left(\mathscr{O}_{W}\left(3 C_{0}+(2 m-1) f\right)\right) \text { if } X \text { is of Type } 4 ; \\
& H^{0}\left(\mathscr{O}_{W}\left(3 C_{0}+3 f\right)\right) \text { if } X \text { is of Type } 5.1 ; \text { and } \\
& H^{0}\left(\mathscr{O}_{W}\left(3 C_{0}+3 m f\right)\right) \oplus H^{0}\left(\mathscr{O}_{W}\left(3 C_{0}+(2 m-2) f\right)\right) \text { if } X \text { is of Type } 5.2 .
\end{aligned}
$$

By Lemma 2.5(e), $\gamma_{2}$ in fact surjects onto $B$, so the cokernel of $\gamma_{2}$ is

$$
\begin{aligned}
& H^{0}\left(\mathscr{O}_{W}((2 m-2) f)\right) \text { if } X \text { is of Type } 3.2 ; \\
& H^{0}\left(\mathscr{O}_{W}((3 m-1) f) \oplus H^{0}\left(\mathscr{O}_{W}((2 m-2) f)\right) \text { if } X \text { is of Type } 4 ;\right. \\
& H^{0}\left(\mathscr{O}_{W}\left(3 C_{0}\right)\right) \oplus H^{0}\left(\mathscr{O}_{W}(3 f) \oplus H^{0}\left(\mathscr{O}_{W}\right) \text { if } X \text { is of Type } 5.1 ;\right. \text { and } \\
& H^{0}\left(\mathscr{O}_{W}(3 m f)\right) \oplus H^{0}\left(\mathscr{O}_{W}((2 m-2) f)\right) \text { if } X \text { is of Type } 5.2 .
\end{aligned}
$$

Then the dimension of the cokernel of $\gamma_{2}$ is

$2 m-1$ if $X$ is of Type 3.2;

$5 m-1$ if $X$ is of Type 4 ;

9 if $X$ is of Type 5.1 ; and

$5 m$ if $X$ is of Type 5.2.

Recall that $2 m$ is the degree of $W$, and $W$ is a surface of minimal degree, so $2 m=p_{g}(X)-2$; therefore the number of extra generators of $R$ in degree 3 is $p_{g}(X)-3$ if $X$ is of Type 3.2.

Finally from (5.2.1), the splitting (2.1.1) of $H^{0}\left(\varphi_{*} \mathscr{O}_{X}\left(n K_{X}\right)\right)$ has four nonzero summands if $n \geq 3$. Then the splitting of $\gamma_{n}$ in summands according to the algebra structure of $\varphi_{*} \mathscr{O}_{X}$ and Lemma 2.5(e) imply the surjectivity of $\gamma_{n}$ for all $n \geq 3$.

Remark 5.3. It is a common phenomenon that, if a graded ring $R$ is generated in degree less than or equal to 2, then the Veronese 2-subring $R^{\prime}$ of $R$ is generated in degree 1. Theorems 2.4, 2.6, 2.7 and 5.2 show the existence of several families of surfaces $X$ of general type (both regular and irregular; precisely, surfaces $X$ of Types 1, 2 and 3.2) such that the Veronese 2-subring of their canonical ring is generated in degree 1, despite the fact that their canonical ring is not generated in degree less than or equal to 2 .

\section{ACKNOWLEDGEMENTS}

We thank Dale Cutkosky and N. Mohan Kumar for some very valuable discussions and comments.

\section{REFERENCES}

[Bad01] L. Bădescu, Algebraic surfaces, Universitext, Springer-Verlag, New York, 2001. MR 1805816 (2001k:14068)

[BS00] M. Beltrametti and T. Szemberg, On higher order embeddings of Calabi-Yau threefolds, Arch. Math. (Basel) 74 (2000), 221-225. MR.1739501 (2001d:14039)

[Cas06] G. Casnati, Examples of Calabi-Yau threefolds as covers of almost-Fano threefolds, Geom. Dedicata 119 (2006), 169-179. MR2247656 (2007c:14037)

[GP98] F.J. Gallego and B.P. Purnaprajna, Very ampleness and higher syzygies for Calabi-Yau threefolds, Math. Ann. 312 (1998), 133-149. MR1645954(99g:14048)

[GP01] F.J. Gallego and B.P. Purnaprajna, Some results on rational surfaces and Fano varieties, J. Reine Angew. Math. 538 (2001), 25-55. MR1855753(2002f:14024) 
[GP03] F.J. Gallego and B.P. Purnaprajna, On the canonical rings of covers of surfaces of minimal degree, Trans. Amer. Math. Soc. 355 (2003), 2715-2732 MR.1975396|(2004b:14069)

[GP07] F.J. Gallego and B.P. Purnaprajna, Classification of quadruple Galois canonical covers II, J. Algebra 312 (2007), 798-828. MR.2333185|(2008m:14075)

[GP08] F.J. Gallego and B.P. Purnaprajna, Classification of quadruple Galois canonical covers I, Trans. Amer. Math. Soc. 360 (2008), 5489-5507. MR2415082 (2009g:14041)

[Gre82] M. Green, The canonical ring of a variety of general type, Duke Math. J. 49 (1982), 1087-1113. MR683012 (84k:14006)

[Hor76] E. Horikawa, Algebraic surfaces of general type with small $c_{1}^{2}$, I, Ann. of Math. (2) 104 (1976), 357-387. MR0424831 (54:12789)

[Kon91] K. Konno, Algebraic surfaces of general type with $c_{1}^{2}=3 p_{g}-6$, Math. Ann. 290 (1991), 77-107. MR:1107664 (92i:14039)

[Lip69] J. Lipman, Rational singularities, with applications to algebraic surfaces and unique factorization, Inst. Hautes Études Sci. Publ. Math. 36 (1969), 195-279. MR0276239 (43:1986)

[Mum70] D. Mumford, Varieties defined by quadratic equations, Corso CIME in Questions on Algebriac Varieties, Rome (1970), 30-100. MR0282975 (44:209)

[OP95] K. Oguiso and T. Peternell, On polarized canonical Calabi-Yau threefolds, Math. Ann. 301 (1995), 237-248. MR1314586 (96b:14050)

[Xia90] G. Xiao, Degree of the bicanonical map of a surface of general type, Amer. J. Math. 112 (1990), 713-736. MR1073006 (91i:14030)

Departamento de Álgebra, Universidad Complutense de Madrid, Madrid, Spain

E-mail address: gallego@mat.ucm.es

Department of Mathematics, University of Kansas, Lawrence, Kansas 66045-2142

E-mail address: purna@math.ku.edu 